\title{
Host Galaxies, Clustering, Eddington Ratios, and Evolution of Radio, X-Ray, and Infrared-selected AGNs.
}

\section{Citation}

Hickox, Ryan C., Christine Jones, William R. Forman, Stephen S. Murray, Christopher S. Kochanek, Daniel Eisenstein, Buell T. Jannuzi, et al. 2009. "Host Galaxies, Clustering, Eddington Ratios, and Evolution of Radio, X-Ray, and Infrared-selected AGNs." The Astrophysical Journal 696 (1) (April 21): 891-919. doi:10.1088/0004-637x/696/1/891.

\section{Published Version}

doi::10.1088/0004-637X/696/1/891

\section{Permanent link}

http://nrs.harvard.edu/urn-3:HUL.InstRepos:26526400

\section{Terms of Use}

This article was downloaded from Harvard University's DASH repository, and is made available under the terms and conditions applicable to Other Posted Material, as set forth at http:// nrs.harvard.edu/urn-3:HUL.InstRepos:dash.current.terms-of-use\#LAA

\section{Share Your Story}

The Harvard community has made this article openly available.

Please share how this access benefits you. Submit a story.

Accessibility 


\title{
HOST GALAXIES, CLUSTERING, EDDINGTON RATIOS, AND EVOLUTION OF RADIO, X-RAY, AND INFRARED-SELECTED AGNs
}

\author{
Ryan C. Hickox ${ }^{1}$, Christine Jones ${ }^{1}$, William R. Forman ${ }^{1}$, Stephen S. Murray ${ }^{1}$, Christopher S. KochaneK ${ }^{2}$, \\ Daniel Eisenstein ${ }^{3}$, Buell T. Jannuzi ${ }^{4}$, Arjun Dey ${ }^{4}$, Michael J. I. Brown ${ }^{5}$, Daniel Stern ${ }^{6}$, Peter R. Eisenhardt ${ }^{6}$, \\ Varoujan Gorjian $^{6}$, Mark Brodwin ${ }^{1,4}$, Ramesh Narayan ${ }^{1}$, Richard J. Cool ${ }^{7}$, Almus Kenter ${ }^{1}$, Nelson Caldwell ${ }^{1}$, \\ AND Michael E. ANDERSON ${ }^{1,8}$ \\ ${ }^{1}$ Harvard-Smithsonian Center for Astrophysics, 60 Garden Street, Cambridge, MA 02138, USA; rhickox@cfa.harvard.edu

 \\ USA \\ ${ }^{3}$ Steward Observatory, 933 North Cherry Avenue, Tucson, AZ 85721, USA \\ ${ }^{4}$ National Optical Astronomy Observatory, Tucson, AZ 85726-6732, USA \\ ${ }^{5}$ School of Physics, Monash University, Clayton 3800, Victoria, Australia \\ 6 Jet Propulsion Laboratory, California Institute of Technology, Pasadena, CA 91109, USA \\ ${ }^{7}$ Princeton University Observatory, Peyton Hall, Princeton, NJ 08544-1001, USA \\ ${ }^{8}$ Department of Astronomy, California Institute of Technology, Pasadena, CA 91109, USA \\ Received 2008 September 19; accepted 2009 February 2; published 2009 April 20
}

\begin{abstract}
We explore the connection between different classes of active galactic nuclei (AGNs) and the evolution of their host galaxies, by deriving host galaxy properties, clustering, and Eddington ratios of AGNs selected in the radio, Xray, and infrared (IR) wavebands. We study a sample of 585 AGNs at $0.25<z<0.8$ using redshifts from the AGN and Galaxy Evolution Survey (AGES). We select AGNs with observations in the radio at $1.4 \mathrm{GHz}$ from the Westerbork Synthesis Radio Telescope, X-rays from the Chandra XBoötes Survey, and mid-IR from the Spitzer IRAC Shallow Survey. The radio, X-ray, and IR AGN samples show only modest overlap, indicating that to the flux limits of the survey, they represent largely distinct classes of AGNs. We derive host galaxy colors and luminosities, as well as Eddington ratios, for obscured or optically faint AGNs. We also measure the two-point cross-correlation between AGNs and galaxies on scales of $0.3-10 h^{-1} \mathrm{Mpc}$, and derive typical dark matter halo masses. We find that: (1) radio AGNs are mainly found in luminous red sequence galaxies, are strongly clustered (with $M_{\text {halo }} \sim 3 \times 10^{13} h^{-1} M_{\odot}$ ), and have very low Eddington ratios $\lambda \lesssim 10^{-3}$; (2) X-ray-selected AGNs are preferentially found in galaxies that lie in the "green valley" of color-magnitude space and are clustered similar to the typical AGES galaxies $\left(M_{\text {halo }} \sim 10^{13} h^{-1} M_{\odot}\right)$, with $10^{-3} \lesssim \lambda \lesssim 1$; (3) IR AGNs reside in slightly bluer, slightly less luminous galaxies than X-ray AGNs, are weakly clustered ( $M_{\text {halo }} \lesssim$ $10^{12} h^{-1} M_{\odot}$ ), and have $\lambda>10^{-2}$. We interpret these results in terms of a simple model of AGN and galaxy evolution, whereby a "quasar" phase and the growth of the stellar bulge occurs when a galaxy's dark matter halo reaches a critical mass between $\sim 10^{12}$ and $10^{13} M_{\odot}$. After this event, star formation ceases and AGN accretion shifts from radiatively efficient (optical- and IR-bright) to radiatively inefficient (optically faint, radio-bright) modes.
\end{abstract}

Key words: galaxies: active - large-scale structure of universe - quasars: general - radio continuum: galaxies surveys - X-rays: galaxies

Online-only material: color figures

\section{INTRODUCTION}

There is increasing evidence that the evolution of galaxies is related to the evolution of their supermassive black holes (SMBHs), which primarily grow through accretion of material as active galactic nuclei (AGNs). This connection between galaxies and SMBHs is suggested by the observed tight correlation between SMBH and galaxy bulge masses (e.g., Magorrian et al. 1998; Ferrarese \& Merritt 2000; Gebhardt et al. 2000), and the similar redshift evolution of star formation and AGN activity (e.g., Madau et al. 1996; Ueda et al. 2003). Galaxies show a well-established bimodality in color that separates blue, generally disk-dominated, star-forming galaxies (the "blue cloud") from red, generally bulge-dominated galaxies (the "red sequence"; see, e.g., Strateva et al. 2001; Blanton 2006; Faber et al. 2007, and references therein). Recent work has suggested that AGNs may play a crucial role in the origin of this bimodality, and particularly the quenching of star formation in blue galaxies and their transition to the red sequence (e.g.,
Hopkins et al. 2006a; Croton et al. 2006; Khalatyan et al. 2008).

Valuable clues to the associated evolution of galaxies and AGNs come from the properties of AGN hosts. It is wellestablished that radio AGNs are most commonly found in massive early-type galaxies (e.g., Yee \& Green 1987; Best et al. 2005), and that a majority of bright local ellipticals show low-power nuclear radio sources (Sadler et al. 1989). Among optically selected AGNs, Kauffmann et al. (2003a) found that low-redshift, low-luminosity AGNs reside in massive galaxies with generally old stellar populations, and that the stellar population becomes younger for galaxies with increasing [O III] luminosity. At higher redshifts $(0.5 \lesssim z \lesssim 1.5)$, X-rayselected AGNs are also found in massive galaxies with generally red colors (Alonso-Herrero et al. 2008), although some studies have also found an excess of AGNs in the "green valley" between the blue cloud and red sequence, compared to the distribution of quiescent galaxies (Sánchez et al. 2004; Nandra et al. 2007; Georgakakis et al. 2008; Silverman et al. 2008; Kocevski et al. 
2009b; Schawinski et al. 2009b; Treister et al. 2009). The differences in host galaxies between AGNs of different types suggest that the process of nuclear accretion is related to the mass and age of the host galaxy.

Further constraints on the links between AGNs and galaxies come from the clustering of AGNs and their local galaxy environments. Among quiescent galaxies, red galaxies are more strongly clustered, and therefore are found in denser environments, than blue galaxies (for some recent results see, e.g., Zehavi et al. 2005; Coil et al. 2008). Clustering measurements on scales $\gtrsim 1 \mathrm{Mpc}$ allow us to estimate the masses of the dark matter halos in which galaxies reside (e.g., Sheth \& Tormen 1999). There is evidence that the evolution of galaxies is strongly linked to host halo mass (e.g., Faber et al. 2007), and similar relationships may also exist for AGN activity. Previous works have found somewhat differing results for AGN clustering and environments, largely owing to different samples of sources (see Brown et al. 2001, for a summary of previous results). However, a general picture has emerged in which radio-loud AGNs are found in dense environments, and massive halos, similar to galaxy clusters, while radio-quiet AGNs are found in poorer environments similar to field galaxies.

Recently, with large surveys such as the Sloan Digital Sky Survey (SDSS; York et al. 2000), the 2dF QSO Redshift Survey (Croom et al. 2004), and the DEEP2 survey (Davis et al. 2003), AGN-galaxy clustering results have been placed on firmer statistical footing. Studies of the autocorrelation of optically selected quasars from the $2 \mathrm{dF}$ and SDSS showed that their clustering amplitude increases with redshift out to $z \sim 3$, indicating that at all redshifts they inhabit dark matter halos of similar characteristic mass $\sim 3 \times 10^{12} M_{\odot}$ (Porciani et al. 2004; Croom et al. 2005; Coil et al. 2007; Myers et al. 2007; Shen et al. 2007; da Ângela et al. 2008; Padmanabhan et al. 2009). This suggests that luminous quasar activity is related to the mass of the surrounding dark matter halo. Among lower luminosity optically selected AGNs from the SDSS, the Seyfert galaxies are generally less clustered than the full galaxy sample (Kauffmann et al. 2004), but show comparable large-scale clustering to control samples of quiescent galaxies with similar properties to the AGN hosts (Li et al. 2006; Mandelbaum et al. 2009). Very low luminosity AGNs (low ionization nuclear emission regions (LINERs)) show no significant bias relative to normal galaxies $^{9}$ (Miller et al. 2003; Constantin \& Vogeley 2006). At higher redshifts, Coil et al. (2007) found that quasars in the DEEP2 fields at $z \sim 1$ show a slight antibias (although at the 1$2 \sigma$ level) relative to all galaxies, similar to the antibias observed for blue galaxies.

$\mathrm{X}$-ray AGNs are generally strongly clustered and reside in relatively dense environments. Studies of the spatial autocorrelation of X-ray AGNs detected with Chandra and XMMNewton (e.g., Basilakos et al. 2004, Gilli et al. 2005, Yang et al. 2006, Puccetti et al. 2006, Miyaji et al. 2007) generally obtain relatively large clustering amplitudes (although with differing results that may reflect a variation in clustering with X-ray flux; Plionis et al. 2008). Recently, Coil et al. (2009) found that Xray AGNs in the Extended Groth Strip are significantly biased relative to galaxies in the DEEP2 survey, in contrast to the results for optical quasars. Correspondingly, Georgakakis et al. (2007) showed that X-ray AGNs in DEEP2 at $z \sim 1$ generally lie in overdense regions, while in the Extended Chandra Deep Field

\footnotetext{
9 Throughout this paper, the terms "quiescent" or "normal" refer to galaxies with no detected AGN activity, but do include star-forming galaxies.
}

South, Silverman et al. (2008) found that AGNs in "green" hosts at $0.4<z<1.1$ preferentially reside in large-scale structures such as walls or filaments. Other recent works (Lehmer et al. 2009; Kocevski et al. 2009a; Galametz et al. 2009) have found an enhancement of the density of X-ray-selected AGNs in largescale structures at relatively high redshifts $(z \gtrsim 0.9)$. However, the significance of the results for X-ray AGNs has been limited by relatively small AGN samples.

Compared to X-ray-selected sources, radio-selected AGN are found in even denser environments including $X$-ray groups and clusters (e.g., Croston et al.). Radio AGNs show strong spatial clustering similar to local elliptical galaxies (e.g., Mandelbaum et al. 2009; Wake et al. 2008), with evidence for higher amplitude for more powerful sources (e.g., Overzier et al. 2003).

These results indicate that AGNs selected using different techniques represent separate populations, with differences in host galaxies, environments, and accretion modes. Large redshift surveys with extensive multiwavelength coverage now make it possible to explore, within the same uniform data set, (1) host galaxies, (2) clustering, and thus dark matter halo masses, and (3) Eddington ratios of AGNs selected by these different techniques.

In this paper, we study a sample of AGNs in the redshift interval $0.25<z<0.8$, using spectroscopic redshifts from the AGN and Galaxy Evolution Survey (AGES). We focus on AGNs selected in the radio, X-ray, and infrared (IR) bands, which provide relatively efficient and unbiased ways of identifying certain populations of AGNs (for a review see Mushotzky 2004). We do not, in general, use optical spectral diagnostics for selecting AGNs, because of selection effects that can arise from the low-resolution fiber spectroscopy of some of the sources. We place particular emphasis on obscured or optically faint AGNs, for which we can study the optical properties of their host galaxies. For these objects we can also measure host galaxy bulge luminosities and thus estimate black hole masses (assuming an $L_{\mathrm{bul}}-M_{\mathrm{BH}}$ relation), in order to derive Eddington ratios.

This paper is organized as follows. In Section 2, we describe the Boötes multiwavelength data set. In Section 3, we describe the main AGES galaxy sample, and in Section 4, we discuss the samples of radio, X-ray, and IR-selected AGNs. In Section 5, we present results on the colors and luminosities of AGN host galaxies. We describe our correlation analysis in Section 6, and present the results, along with estimates of dark matter halo mass, in Section 7. In Section 8, we calculate average X-ray spectra and Eddington ratios for the different samples of AGNs. In Section 9, we discuss our results in terms of a simple picture of AGN and galaxy evolution, in which luminous quasar activity and the termination of star formation occur at a characteristic dark matter halo mass. We summarize our results in Section 10. We also include two appendices that describe (Appendix A) our correction for AGN contamination of host galaxy colors, and (Appendix B) limits on the contamination of the IR AGN sample by star-forming galaxies.

Throughout this paper we assume a lambda cold dark matter $(\Lambda \mathrm{CDM})$ cosmology with $\Omega_{\mathrm{m}}=0.3$ and $\Omega_{\Lambda}=0.7$. For direct comparison with other works, we assume $H_{0}=70 \mathrm{~km} \mathrm{~s}^{-1}$ $\mathrm{Mpc}^{-1}$, except for comoving distances and absolute magnitudes, which are explicitly given in terms of $h=H_{0} /\left(100 \mathrm{~km} \mathrm{~s}^{-1}\right.$ $\left.\mathrm{Mpc}^{-1}\right)$. The NOAO Deep Wide-Field Survey (NDWFS) photometry in the $B_{W}, R$, and $I$ bands is presented in Vega magnitudes, while magnitudes in the SDSS $u$ and $r$ bands are AB normalized. All quoted uncertainties are $1 \sigma$ (68\% confidence). 


\section{OBSERVATIONS}

The $9 \mathrm{deg}^{2}$ survey region in Boötes covered by the NDWFS (Jannuzi \& Dey 1999) is unique among extragalactic multiwavelength surveys in its wide field and uniform coverage using space- and ground-based observatories, including the Chandra $X$-Ray Observatory and the Spitzer Space Telescope (SST). Extensive optical spectroscopy makes this field especially well suited for studying the statistical properties of a large number of AGNs (C. Kochanek et al. 2009, in preparation). The areas covered by the X-ray, optical, infrared, and radio surveys are shown in Figure 1.

Redshifts for this study come from AGES, which used the Hectospec multifiber spectrograph on the MMT (Fabricant et al. 2005). We use AGES Data Release 2 (DR 2), which consists of all the AGES spectra taken in 2004-2006. The 2004 observations covered 15 pointings for a total area of $7.9 \mathrm{deg}^{2}$, which we define to be the main AGES survey region. In the 2004 observations, targets include (1) all extended sources (i.e., galaxies, see Section 4.4) with $R \leqslant 19.2$ (2) a randomly selected sample of $20 \%$ of all extended sources with $19<R \leqslant 20$, (3) all extended sources with $R \leqslant 20$ and Infrared Array Camera (IRAC) 3.6, 4.5, 5.8, and $8.0 \mu \mathrm{m}$ magnitudes $\leqslant 15.2,15.2,14.7$, and 13.2, respectively. In addition, (4) fainter sources were observed, selected mainly from objects that are counterparts of Chandra X-ray sources (Murray et al. 2005; Brand et al. 2006a; Kenter et al. 2005), radio sources from the Very Large Array (VLA) FIRST survey (Becker et al. 1995), and objects selected from $24 \mu \mathrm{m}$ observations with the Multiband Imaging Photometer (MIPS) for Spitzer (E. Le Floc'h et al. 2009 , in preparation). AGES DR 2 contains $I$-selected targets with $I \leqslant 21.5$ for point sources and $I \leqslant 20.5$ for extended sources.

AGES redshifts and spectral classifications follow the procedures used by SDSS. Redshifts are measured by comparing the extracted spectroscopy to the grid of galaxy templates, and choosing the combination of template and redshift that minimizes the $\chi^{2}$ between the data and the model. Good redshifts are defined to be those that do not have a second minimum in $\chi^{2}$ that is within 0.01 in reduced $\chi^{2}$ to the best-fit value. All redshifts were examined by eye and bad fits were flagged manually.

Optical photometry from NDWFS was used for the selection of AGES targets and to derive optical colors and fluxes for AGES sources. NDWFS images were obtained with the Mosaic1 camera on the $4 \mathrm{~m}$ Mayall Telescope at Kitt Peak National Observatory, with $50 \%$ completeness limits of 26.7, 25.0, and $24.9 \mathrm{mag}$, in the $B_{W}, R$, and $I$ bands, respectively. Photometry is derived using SExtractor (Bertin \& Arnouts 1996).

Radio data are taken from the Westerbork Synthesis Radio Telescope (WSRT) $1.4 \mathrm{GHz}$ radio survey that covers $\approx 7 \mathrm{deg}^{2}$ in the center of the NDWFS field (de Vries et al. 2002). 3035 radio sources are detected in the AGES survey region, to a limiting flux of $\approx 0.1 \mathrm{mJy}$ and beam size $13^{\prime \prime} \times 27^{\prime \prime}$. Centroid positions of the radio sources are measured to 0.'4.

$\mathrm{X}$-ray data are taken from the XBoötes survey, which covers the full AGES spectroscopic region. The XBoötes mosaic consists of $1265 \mathrm{ks}$ Chandra ACIS-I exposures and is the largest contiguous field observed to date with Chandra (Murray et al. 2005). Owing to the shallow exposures and low background in the ACIS detector, X-ray sources can be detected to high significance with as few as four counts. The XBoötes catalog contains 3293 X-ray point sources with four or more counts
(Kenter et al. 2005), and of these, 2724 lie in the AGES survey region.

Mid-IR observations are taken from the Spitzer IRAC Shallow Survey (Eisenhardt et al. 2004), which covers the full AGES field in all four IRAC bands $(3.6,4.5,5.8$, and $8 \mu \mathrm{m}$ ), with $5 \sigma$ flux limits of $6.4,8.8,51$, and $50 \mu \mathrm{Jy}$ respectively. The IRAC photometry is described in detail in Brodwin et al. (2006). The catalog contains 15,488 sources detected in all four IRAC bands, and of these, 14,069 lie within the AGES survey region.

\section{GALAXY SAMPLE}

The first step in our analysis is to define a statistical sample of galaxies from the AGES catalog. These galaxies will be used to provide a comparison sample against which we can compare the properties of AGN host galaxies. These galaxies will also be used to determine the spatial cross-correlation between AGNs and normal galaxies, and so to derive AGN clustering.

The statistical galaxy sample consists of AGES galaxy targets with the flux limit $I<20$. We outline the key aspects of the statistical galaxy sample here, while full details will be presented by D. Eisenstein et al. (2009, in preparation). AGES galaxy targets must satisfy quality cuts in the $I$ band and either the $B_{W}$ and $R$ bands in the NDWFS imaging. In addition, all objects in the sample must have good detections (SExtractor FLAGS $<8$ ) in all three bands, to allow for $K$ corrections (this eliminates very few sources). In addition, objects must be detected as an extended source (see Section 4.4) in at least one of the three bands.

In this analysis, we use the auto (Kron) I-band magnitude; however, in some regions the $I$-band photometry suffers from low-surface-brightness halos around bright stars, which causes the Kron galaxy magnitudes to be overly bright. To correct for this, we define the $I_{R}$ magnitude constructed from the sum of the $R$ band Kron magnitude and the $R-I$ color derived from $6^{\prime \prime}$ aperture magnitudes. We then compare $I_{R}$ and the $I$ (Kron) magnitude and compute $I_{\text {tot }}$, which consists of the fainter magnitude (if the two differ significantly) and otherwise is the average of the two magnitudes. $I_{\text {tot }}$ is typically $0.01-$ 0.09 mag fainter than the $I$ (Kron) magnitude. We cut the sample at $I_{\text {tot }}<19.95$, which excludes $2 \%$ of the galaxies with Kron magnitude $I<20$. The positions on the sky of the AGES galaxies with $0.25<z<0.8$ are shown in Figure 2, and their redshift distribution is shown in Figure 3.

\subsection{Sampling Weights}

For the targets that pass the above criteria, a complicated set of random sparse sampling criteria were used to select objects for observation. In general, $100 \%$ of bright $(I<18.5)$ galaxies were observed, while the sampling rate for sources with $18.5<I<20$ was $20 \%-30 \%$ (depending on whether they pass flux cuts in interesting UV, optical, or IR bands). In addition, many galaxies were assigned fibers (and obtained redshifts) as part of the AGES observations, but were not systematically targeted. Some of these redshifts correspond to X-ray or radioselected AGNs and so are used in this analysis. However, these objects are not included in the "main" statistical galaxy sample that we use for AGN-galaxy cross-correlation. The main AGES sample includes only those galaxies that were systematically targeted in the random sampling.

For each target in the main AGES sample that has a welldetermined redshift, we assign a series of sampling weights. 
First, we assign a sparse sampling weight, equal to the inverse of the random sampling rate that is known exactly from the target selection (that is, if $20 \%$ of the objects in a class are targeted, the sampling weight is 5). Second, we determine a target assignment weight, given by the inverse of the probability that a target was assigned a fiber in the 2004-2006 observing runs. Owing to the high completeness of the AGES observing strategy for the main galaxy sample, this fiber weight averages only 1.05. Finally, we assign a redshift weight, given by the inverse probability that an object that is assigned to a fiber succeeds in yielding a well determined redshift. We determine this weight for various subsamples of the targets in bins of magnitude, surface brightness, color, and sparse sampling class, by computing the ratio of the number of attempts to the number of well determined redshifts. The total statistical weight $W$ associated with each galaxy is the product of these three weights.

\subsection{Bright Star Exclusion}

Owing to difficulties in obtaining redshifts for objects in the vicinity of bright stars, objects around bright sources were not targeted for AGES spectroscopy. The exclusion was performed using a bright source list taken from objects in the USNOB catalog (Monet et al. 2003) with $R<17$. A number of these bright "stars" correspond to extended sources, some of which are AGES target galaxies. To minimize the exclusion of targets around AGES galaxies, the 475 objects that match extended sources in the Two Micron All Sky Survey (2MASS) catalog (Skrutskie et al. 2006) were removed from the bright star list before the AGES targets were selected. This leaves 6690 bright sources around which objects were not targeted for AGES spectroscopy. Possible target objects were excluded around these bright sources, with an exclusion radius that varied with the flux of the bright source and the flux of the target object. Around a bright source of magnitude $R$, faint target objects were excluded out to a radius

$$
r_{\text {limit }}=20^{\prime \prime}+5^{\prime \prime}(15-R),
$$

while brighter target objects were excluded inside smaller radii.

To minimize biases in our spatial clustering analysis, we avoid the regions on the sky from which AGES targets were excluded. For simplicity, we remove from our sample all AGES sources (regardless of flux) within $r_{\text {limit }}$ of a bright source, including the bright source itself if it is an AGES galaxy. We exclude the same regions in our random catalog (Section 3.4). This exclusion removes 776 AGES target galaxies that correspond to the objects in the bright source catalog, but were not matched to 2MASS extended sources. However, all but 19 of these bright AGES targets lie at $z<0.25$. To minimize the effects of removing the bright AGES galaxies, we restrict our analysis to objects at $z>0.25$, which leaves us with 6262 galaxies, with total statistical weight equal to 15,653 . We refer to these galaxies as the "main" AGES statistical sample and focus on this sample in our analysis.

\subsection{K-Corrections and Colors}

One powerful diagnostic for understanding the properties of galaxies is their distribution in color and absolute magnitude. Many previous studies have found that the color-magnitude distribution of galaxies is bimodal, separated into a red sequence of luminous, bulge-dominated, passively evolving galaxies, and a blue cloud of less luminous, star-forming, disk-dominated galaxies (e.g., Strateva et al. 2001; Blanton et al. 2003b; Kauffmann et al. 2003c).

For the AGES galaxies, we use the $4^{\prime \prime}$ aperture magnitudes and $I_{\text {tot }}$ to perform $K$-corrections and thus derive the restframe colors and absolute magnitudes. As discussed above, only galaxies with good $B_{W}, R$, and $I$ photometry are included in the galaxy sample. We obtain $B_{W}$ and $R$ magnitudes by adding the $B_{W}-I$ and $R-I 4^{\prime \prime}$ aperture colors, respectively, to $I_{\text {tot }}$. These magnitudes are in general close to the Kron magnitudes; the mean and standard deviation in $\Delta m=m_{\text {tot }}$ $m_{\text {Kron }}$ are $(0.01,0.10)$ and $(-0.003,0.038)$ in the $B_{W}$ and $R$ bands, respectively. We convert Vega magnitudes to AB using corrections of $+0.02 \mathrm{mag},+0.19 \mathrm{mag}$, and $+0.44 \mathrm{mag}$ for the $B_{W}$, $R$, and $I$ bands, respectively, and correct absolute magnitudes for interstellar extinction (Schlegel et al. 1998), which for this field is only $A_{I}=0.02 \mathrm{mag}$.

We define rest-frame colors and absolute magnitudes in terms of photometric bands that are close to the wavelengths probed by the NDWFS photometry at typical AGES redshifts. We convert the $I$ and $B_{W}$ magnitudes to ${ }^{0.1} r$ and ${ }^{0.1} u$, respectively, which are defined as the AB magnitudes in the SDSS $r$ and $u$ bands, shifted blueward by $z=0.1$. These conversions are insensitive to the galaxy spectral energy distribution (SED) at $z=0.42$ and 0.27 , respectively. Calculating rest-frame colors in terms of these bands allows for straightforward comparison to results from SDSS (e.g., Blanton et al. 2003a, 2003b, 2003c; Kauffmann et al. 2003b). The $K$-corrections are performed using kcorrect version 3.2 (Blanton et al. 2003a); using an updated version of kcorrect (ver. 4.1.4) produces no significant change in the $K$-corrections.

In addition to calculating rest-frame colors for each galaxy, we derive the absolute magnitude $\left(M_{0.1}\right)$ in the ${ }^{0.1} r$ band at $z=0.1$. We also calculate the absolute magnitude corrected for passive evolution, assuming $1.6 \mathrm{mag}$ of evolution per unit redshift relative to the observed redshift (Eisenstein et al. 2005). This correction varies from 0 at $z=0.1$ (by definition) to +1.1 mag at $z=0.8$, in the sense that the evolution-corrected absolute magnitude at $z=0.1$ is fainter than the observed $M_{0.1}$. The evolution-corrected absolute magnitudes are used only in the estimates of galaxy bulge and black hole masses (Section 8.2); elsewhere we use absolute magnitudes that are not corrected for evolution.

Figure 4 shows rest-frame ${ }^{0.1}(u-r)$ color versus $M_{0.1}$ for galaxies in the AGES main sample in the redshift interval $0.25<z<0.8$. The bimodal distribution in galaxy color (the red sequence and blue cloud) is clearly evident. We divide the galaxies into red and blue subsamples; to account for the slope of the red sequence, we define the quantity

$$
A={ }^{0.1}(u-r)+0.08\left(M_{0.1} r+20\right),
$$

(where $M_{0.1_{r}}$ is calculated for $h=1$ ) and divide red and blue galaxies by a cut in $A$. The red sequence evolves slightly in color with redshift, so we empirically determine the median value in $A$ for the red sequence as a function of $z$; this varies from $A_{\text {red }}=2.64$ at $z=0.25$ to $A_{\text {red }}=2.52$ at $z=0.8$. At each redshift, we set the boundary between red and blue samples to be 0.3 mag blueward of $A_{\text {red }}$. The selection boundaries for $z=0.25$ and $z=0.8$ are shown in Figure 4. This criterion selects 3119 red galaxies and 3143 blue galaxies in the redshift interval $0.25<z<0.8$. After a small correction for nuclear contamination from AGNs (Appendix A), we select 3146 red galaxies and 3119 blue galaxies. 




Figure 1. Map of the Boötes survey region, showing the approximate areas covered by the AGES, WSRT (radio), XBoötes (X-ray), and IRAC Shallow Survey (mid-IR) observations.

(A color version of this figure is available in the online journal.)

\subsection{Random Galaxy Catalog}

To derive the spatial correlation of galaxies and AGNs (Section 6) requires an estimate of the number of galaxy-galaxy or AGN-galaxy pairs expected if the galaxies were randomly distributed in comoving space. We therefore produce a catalog of galaxies that are spatially uncorrelated, but reflect the selection function of the AGES sample. To ensure that the fractional uncertainties of the AGN-random catalog pairs are negligible, the density of random galaxies must be significantly larger than in the data catalog. This is especially true on small scales (less than $1 h^{-1} \mathrm{Mpc}$ ), where real galaxies are most strongly clustered. We design the random catalog to contain 200 times the (statistically weighted) number of galaxies in the data catalog.

For each galaxy in the AGES statistical sample with statistical weight $W^{i}$, we generate $N_{i}$ duplicate galaxies, where $N_{i}=$ $200 W^{i}$, rounded to the nearest integer. We then assign each of these random galaxies a statistical weight equal to $W_{R}^{i}=$ $W^{i} / N_{i}$, so that the total statistical weight in the random sample is equal to the total weight in the data.

We place each random galaxy in a random sky position inside the main AGES field (and outside the bright star masks) and offset its redshift by a random $-0.05<\Delta z<+0.05$. This effectively applies a boxcar smoothing to the line of sight distances on scales of 200-300 $\mathrm{h}^{-1} \mathrm{Mpc}$, larger than the largest observed structures, and so eliminates any significant correlation along the line of sight. The redshift distribution of the random catalog is shown in Figure 3, along with the distribution for the observed galaxies.

\section{AGN SAMPLE}

We next define the samples of AGNs for which we will determine host galaxy, clustering, and accretion properties. For complete as possible an AGN census, we select AGNs independently in the radio, X-ray, and IR wavebands. As discussed above, we do not use optical spectroscopic selection in order to avoid systematic effects in the analysis of the fiber spectroscopy. We restrict ourselves to sources that have redshifts from AGES in the interval $0.25<z<0.8$, are located in the



Figure 2. Positions of 6262 AGES galaxies in the main statistical sample (Section 3) at redshifts $0.25<z<0.8$. Points are color-coded by redshift. The black line shows the boundary of the 15 main AGES pointings.

(A color version of this figure is available in the online journal.)



Figure 3. Redshift distribution for galaxies in the main AGES sample and for the AGES random catalog (see Section 3.4). The solid and dotted black histograms show the redshift distribution for AGES main sample galaxies (in our analysis we only include objects at $0.25<z<0.8$, shown by the solid black histogram; for completeness the dotted black histogram shows AGES main sample galaxies at $z<0.25$ ). The red dashed histogram shows the redshift distribution for the random galaxy catalog. For clarity, the histogram values for the random galaxies are divided by 200 , since the random sample contains 200 times as many objects as the (statistically weighted) observed galaxy sample. Note that the observed galaxy histogram is not normalized by statistical weights.

(A color version of this figure is available in the online journal.)

main AGES field, and are away from bright stars, as described in Section 3. In this section, we describe the selection criteria and AGN samples.

\subsection{Radio AGNs}

We first select AGNs in the radio. The AGES catalog contains 525 matches (within $2^{\prime \prime}$ ) to WSRT radio sources, of which 245 are in the AGES field and in the interval $0.25<z<0.8$ (note that the WSRT field is $\approx 7 \mathrm{deg}^{2}$ and does not cover the full AGES 

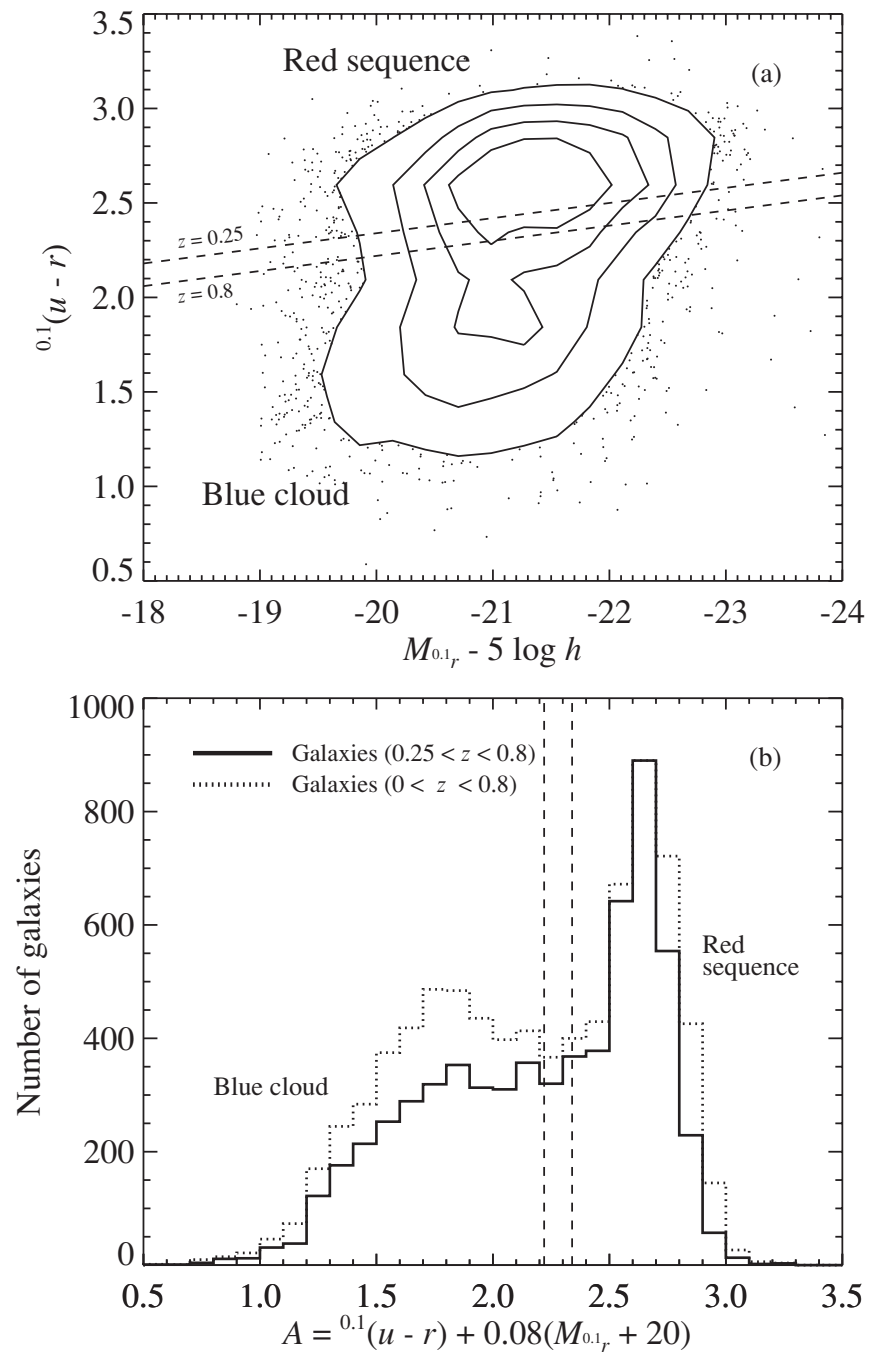

Figure 4. (a) Rest-frame optical luminosities and colors for AGES galaxies. The contours and points show the distribution of rest-frame ${ }^{0.1}(u-r)$ color vs. $M_{0.1}$ absolute magnitude (not corrected for passive evolution), for AGES galaxies in the statistical sample in the redshift interval $0.25<z<0.8$ (points are left out in the region covered by contours). The diagonal dashed lines show the empirical division between red and blue galaxy samples for $z=0.25$ (top) and $z=0.8$ (bottom). (b) Distribution in the color parameter $A$ (see Equation (2)) for AGES galaxies at $0.25<z<0.8$, showing the "red sequence" and "blue cloud." The boundaries between red and blue galaxies at $z=0.25$ and $z=0.8$ are shown as dashed lines, as in (a). The dotted line shows the distribution including galaxies at $z<0.25$, which include many lower luminosity blue galaxies, and which more clearly shows the "green valley."

spectroscopic region, as shown in Figure 1). None of the radio sources match more than one AGES galaxy.

For each radio source we calculate the radio power $P_{1.4 \mathrm{GHz}}$ (in $\mathrm{W} \mathrm{Hz}^{-1}$ ). To obtain the rest-frame power we include a small $K$-correction of $0.1 \mathrm{dex}$, appropriate for the average redshift of the radio-detected objects between $0.25<z<0.8$, and the typical spectrum of faint $1.4 \mathrm{GHz}$ sources $(\alpha \approx 0.5$, where $S_{v} \propto v^{-\alpha}$; Prandoni et al. 2006). The distributions in $P_{1.4 \mathrm{GHz}}$ and redshift are shown in Figure 6(a). For objects with low radio power, the observed emission can be powered by either AGN activity or star formation. It is possible to separate AGNs from star-forming galaxies based on a combination of $P_{1.4 \mathrm{GHz}}$ and optical spectral features such as $\mathrm{H} \alpha$ luminosity (e.g., Kauffmann et al. 2008); however, the required fits to the optical spectra for the AGES galaxies are not currently available. To minimize the number of star-forming galaxies, we restrict our sample



Figure 5. Positions of 585 AGNs in the main AGES field. We show AGNs selected in the radio (orange circles), X-rays (green stars), and IR (red squares) that lie in the main AGES field and away from bright stars, and in the redshift interval $0.25<z<0.8$. The solid line shows the boundary of the main AGES field.

(A color version of this figure is available in the online journal.)

to the 122 sources with $\log P_{1.4 \mathrm{GHz}}>23.8$. In low-redshift samples, almost all galaxies with radio power above this cut are AGNs (see Figure 1 of Kauffmann et al. 2008). ${ }^{10}$ All the radio AGNs in our sample have $P_{1.4 \mathrm{GHz}}<2 \times 10^{26} \mathrm{~W} \mathrm{~Hz}^{-1}$, and so are in the luminosity range typical of Fanaroff \& Riley (1974) type I sources (Ledlow \& Owen 1996). The positions on the sky of the radio AGNs are shown in Figure 5. To test for spurious matches of the radio sources to AGES galaxies, we offset the radio source positions by $1^{\prime}$ and reperformed the source matching. We find only four matches, for which we calculated $P_{1.4 \mathrm{GHz}}$ corresponding to the redshift of the matched AGES galaxy. None of the matches have $\log P_{1.4 \mathrm{GHz}}>23.8$, indicating that there is minimal contamination from spurious matches in our radio AGN sample.

\section{2. $X$-ray AGNs}

We next define the X-ray AGN sample. Among the 3293 Xray sources with $\geqslant 4$ X-ray counts in the Kenter et al. (2005) catalog, 362 of these lie within the AGES field, are not close to bright stars (see Section 3.2), and are matched within 3".5 to objects with good AGES redshifts at $0.25<z<0.8$. We have excluded four objects that show evidence for extended Xray emission that could arise from galaxy groups or clusters (we verify explicitly that this does not significantly affect the clustering results). Nine of the 362 sources in our X-ray AGN sample have two AGES galaxies within 3".5; for these we selected the closer galaxy as the optical counterpart. We test for spurious matches by offsetting the IR-selected AGN positions by $1^{\prime}$ and reperforming the source matching. We find five matches, indicating contamination from spurious matches of only $\approx 1 \%$.

${ }^{10}$ Note that Kauffmann et al. (2008) assume $H_{0}=50 \mathrm{~km} \mathrm{~s}^{-1} \mathrm{Mpc}^{-1}$; our cut is scaled to our assumed cosmology. 



Figure 6. Distribution in redshift and luminosity for (a) radio, (b) X-ray, and (c) IR AGNs with AGES redshifts, in the main AGES field and away from bright stars. Luminosities are the observed $1.4 \mathrm{GHz}$ radio power $\left(P_{1.4 \mathrm{GHz}}\right)$, the $0.5-7 \mathrm{keV}$ luminosity $\left(L_{X}\right)$, and $v L_{v}$ at $4.5 \mu \mathrm{m}$ in the rest frame $\left(L_{4.5} \mu \mathrm{m}\right)$. We focus on the redshift interval $0.25<z<0.8$, shown by the vertical dotted lines, and in the radio, sources with $\log P_{1.4 \mathrm{GHz}}>23.8$ (see Section 4), shown by a horizontal line. Objects included in the analyses are shown by filled symbols. The dashed lines show approximate luminosity limits for the survey flux limits (note that the $\mathrm{X}$-ray limit is for an X-ray spectrum with photon index $\Gamma=1.8$ ). The histograms show the distribution in $z$ and luminosity for sources in this redshift interval. For the radio sources, the dashed histograms show the distributions in $z$ and $P_{1.4 \mathrm{GHz}}$ for the complete sample of radio sources in the interval $0.25<z<0.8$, while the solid histograms show those sources with $\log P_{1.4 \mathrm{GHz}}>23.8$.

(A color version of this figure is available in the online journal.)

For each X-ray source we calculate an $0.5-7 \mathrm{keV}$ luminosity from the number of detected source counts in the $0.5-2 \mathrm{keV}$ and $2-7 \mathrm{keV}$ bands separately, and assuming a power-law X-ray spectrum with photon index $\Gamma=1.8$, typical of AGNs (Tozzi et al. 2006). The corresponding conversions from count rates (in counts s${ }^{-1}$ ) to flux (in erg cm${ }^{-2} \mathrm{~s}^{-1}$ ) are $5.9 \times 10^{-12} \mathrm{ergs} \mathrm{cm}^{-2}$ count ${ }^{-1}$ in the $0.5-2 \mathrm{keV}$ band and $1.9 \times 10^{-11} \mathrm{erg} \mathrm{cm}^{-2}$ count $^{-1}$ in the $2-7 \mathrm{keV}$ band (Kenter et al. 2005). For simplicity we do not include $K$-corrections, which are generally small $(<5 \%)$; for most sources uncertainty in the flux and $\Gamma$ are significantly larger.

The X-ray AGN positions are shown in Figure 5, and their distribution in luminosity and redshift is shown in Figure 6(b). The luminosities of these sources are in the range $10^{42}<L_{X}<$ $10^{45} \mathrm{erg} \mathrm{s}^{-1}$, characteristic of moderate- to high-luminosity AGNs and significantly larger than is typically found for starforming galaxies (e.g., Ranalli et al. 2003). We are therefore confident that the X-ray emission from these objects is powered by nuclear accretion rather than star formation.

We note that since XBoötes sources have as few as 4 counts in $5 \mathrm{ks}$, the fluxes of the faintest sources are affected by the Eddington bias. For a differential flux distribution with $d N / d S \propto S^{-\beta}$, the true mean flux for a source observed with $n$ counts is approximately $n-\beta$ (Eddington 1913). At the XBoötes flux limits $\left(S_{0.5-2 \mathrm{keV}} \sim 5 \times 10^{-15} \mathrm{erg} \mathrm{s}^{-1}\right), \beta \simeq 2$ (Vikhlinin et al. 1995; Kenter et al. 2005), so a source with 4 counts has a true mean flux of $\simeq 2$ counts (Kenter et al. 2005). This correction is relatively small for most sources, and does not affect our interpretation of the X-ray sources as accretiondominated. Correcting for the Eddington bias, all of the sources have $L_{X}>10^{42} \mathrm{erg} \mathrm{s}^{-1}$, so we conclude that contamination by starburst-dominated galaxies will be small. In what follows we use $L_{\mathrm{X}}$ values that are corrected for Eddington bias.

\subsection{IR AGNs}

We finally select AGNs using IRAC observations. With the launch of Spitzer, the mid-IR has provided a sensitive new window for identifying AGNs. The mid-IR SEDs of broadline quasars and Seyfert galaxies typically show a featureless, roughly power-law continuum that rises to longer wavelengths (e.g., Glikman et al. 2006). This SED can be used to separate AGNs from normal and starburst galaxies, whose SEDs exhibit a stellar bump at $1.6 \mu \mathrm{m}$ and then fall at longer wavelengths. Several criteria have been developed to select AGNs based on IRAC colors (Lacy et al. 2004; Stern et al. 2005) or SED fitting (e.g., Alonso-Herrero et al. 2006; Donley et al. 2007). In this 




Figure 7. IRAC color-color diagram for all AGES sources at $0.25<z<0.8$ that lie inside the main AGES field and away from bright stars, and have $5 \sigma$ detections in all four IRAC bands. The dashed line shows the selection region defined by Stern et al. (2005). Symbols for radio and X-ray AGNs are as in Figure 5. Most radio AGNs are found to the lower left, in the region occupied by stars and quiescent galaxies (Stern et al. 2005), while X-ray AGNs are found throughout the distribution, with a significant number in the IRAC AGN selection region.

(A color version of this figure is available in the online journal.)

paper, we use the color selection of Stern et al. (2005), which was derived using the Boötes multiwavelength data and so is well matched to the depth of the survey. Because mid-IR wavelengths are not as strongly affected by dust extinction as the optical or UV, IRAC selection is particularly useful for identifying dustobscured AGNs (e.g., Stern et al. 2005; Rowan-Robinson et al. 2005; Polletta et al. 2006; Hickox et al. 2007).

The Stern et al. (2005) AGN color-color criteria requires that a source be detected in all four IRAC bands. Of the IRAC sources detected in all four bands, 2952 are matched (within 3".5) to AGES sources at $0.25<z<0.8$. The distribution in IRAC colors for these objects are shown in Figure 7. The objects in the bottom left of this diagram have IRAC colors similar to stars $([5.8]-[8.0]<0.8$ and [3.6] $-[4.5]<0.3)$; these are generally passively evolving galaxies that are dominated by starlight in the mid-IR. The diagram also shows a continuous sequence of sources with redder [5.8] - [8.0] and [3.6] - [4.5] colors, which are generally star-forming galaxies with dust features (mainly due to polycyclic aromatic hydrocarbons (PAHs)) that move through the IRAC bands with redshift (see Figure 1 of Stern et al. 2005, for evolutionary tracks of star-forming galaxies on this diagram). A separate sequence of sources with very red [3.6] - [4.5] colors is selected by the Stern et al. (2005) AGN criteria (shown by the dashed line). These SEDs are inconsistent with typical emission from dust heated by star formation, and are characteristic of broad-line quasars and Seyfert galaxies.

Of the AGES sources at $0.25<z<0.8$ matched to the IRAC photometry, these criteria select 238 objects. Six of these sources have two AGES galaxies within 3".5; we selected the closer galaxy as the optical counterpart. We test for spurious matches by offsetting the IR-selected AGN positions by $1^{\prime}$ and find six matches, indicating contamination from spurious matches of only $\approx 2 \%$. The positions of the IR-selected AGNs are shown in Figure 5. For each IRselected AGN, we calculate the rest-frame luminosity $\left(v L_{v}\right)$ at $4.5 \mu \mathrm{m}, L_{4.5} \mu \mathrm{m}$. The SED at rest-frame $4.5 \mu \mathrm{m}$ is probed by the IRAC photometry at all redshifts in our sample, and since this wavelength is significantly redward of the $1.6 \mu \mathrm{m}$ peak in the stellar emission from galaxies, the observed flux is likely dominated by the AGN. We estimate $L_{4.5} \mu \mathrm{m}$ by logarithmically interpolating between the observed $\nu L_{v}$ in the $4.5 \mu \mathrm{m}, 5.8 \mu \mathrm{m}$, and $8 \mu \mathrm{m}$ IRAC bands. The distribution of the IRAC-selected AGNs in $L_{4.5 \mu \mathrm{m}}$ and redshift is shown in Figure 6(c).

One possible limitation of IRAC color selection is in reliability. For the radio and X-ray samples, the high radio and X-ray luminosities are robust indicators of the presence of nuclear accretion. The reliability of IRAC selection is less well studied (although there are discussions of this in Barmby et al. 2006 and Gorjian et al. 2008). The most likely contaminants are starforming galaxies, which in some extreme cases, can have IR luminosities and colors similar to our IR-selected AGN sample.

Our sample of AGNs with AGES spectroscopy appears to suffer little contamination from star-forming galaxies, owing primarily to the relatively bright optical and IRAC flux limits. Donley et al. (2008) examined Spitzer $24 \mu \mathrm{m}$ sources in the GOODS-S field, and found that IRAC color-selected samples in deep surveys have significant contamination from star-forming galaxies, but this contamination decreases for higher IR and optical fluxes. Donley et al. (2008) argue that the selection of AGNs by fitting a power-law SED to the observed IRAC emission reduces the contamination in their sample. To check whether power-law selection would improve the reliability of our sample, we apply the power-law criteria of Donley et al. (2008) to objects with AGES spectra at $0.25<z<0.8$ and detections in all four IRAC bands. We identify 54 power-law AGNs, of which all but one are also selected using the Stern et al. (2005) color selection. Among the power-law AGNs, 53 lie in the X-ray field, and $21(40 \%)$ are detected in X-rays, indicating that they are unambiguously AGNs. In comparison, 184 colorselected AGNs are not selected by the power-law criteria and have X-ray coverage, and of these 97 (53\%) are detected in Xrays. Therefore, in our sample, the IRAC color criteria are equally or more likely to identify X-ray-detected AGNs as the power-law criteria. We conclude that for our relatively shallow IRAC observations and bright optical spectroscopic flux limit, the Stern et al. (2005) color criteria are as reliable as power-law criteria in selecting AGNs.

As another check, we carefully analyze the average $\mathrm{X}$ ray emission from the IRAC color-selected sources that are not detected in X-rays. The average fluxes, stacked spectrum, and distribution of observed X-ray counts indicate that starforming contamination in our IR-selected AGN sample is at most $20 \%$, and most likely $<10 \%$ (see Appendix B for a detailed discussion). We conclude that contamination of the IRAC AGN sample from star-forming galaxies is small.

\subsection{Optical Counterparts}

Useful observational clues as to the nature of AGNs come from the morphologies of their optical counterparts. Objects that are unresolved in the NDWFS images are generally dominated in the optical by the active nucleus, while objects that are optically extended are dominated by their host galaxies and have optically faint or obscured nuclei. For optically extended sources, we can determine the optical properties of the host galaxies, and indirectly, estimate their central black hole masses. We therefore divide the AGN sample into two categories 
based on whether their optical counterparts are extended or unresolved.

The sample of AGNs with extended counterparts is defined as sources having the SExtractor parameter CLASS_STAR $<0.8$ in all of the $B_{W}, R$, and $I$ bands. $92 \%, 97 \%$, and $99 \%$ of these objects have CLASS_STAR $<0.3$ in the $B_{W}, R$, and $I$ bands, respectively. These will be referred to as AGNs with "galaxy" (or "extended") counterparts. Almost all (113) of the radio AGNs, roughly two-thirds (238) of the X-ray AGNs, and half (133) of the IR AGNs have extended optical counterparts. For simplicity, and to obtain the best possible statistics, we include all the optically extended sources in our analysis. We note that, 74, 157, and 111, of the radio, X-ray, and IR AGNs, respectively, were systematically targeted by AGES and are included in the main galaxy sample. We verify explicitly that if we limit the sample only to these sources (which may suffer less complicated selection effects), there is no significant change to our results.

Although the optical counterparts of these sources are extended, the derivation of host galaxy properties is complicated by the fact that an optically weak AGN can still affect the integrated colors from the galaxy. By comparing the aperture photometry for these AGNs to that of normal galaxies, we carefully estimate the AGN color contamination and derive corrections to the host galaxy colors. Details of these corrections are given in Appendix A. The color corrections are small (typically $\simeq 0.1 \mathrm{mag}$ ), and do not significantly affect our conclusions.

The second category of AGN optical counterparts are those with pointlike optical morphologies. We define pointlike sources as those with CLASS_STAR $\geqslant 0.8$ in any of the $B_{W}, R$, and $I$ bands. The flux from most of these sources is dominated by nuclear emission in the $B_{W}$; for simplicity, we will refer to them as "unresolved," but we note that many of these sources are extended in the $R$ and $I$ bands in which there is a larger contribution from the host galaxy. Although these objects are dominated by the nucleus at blue optical wavelengths, they would not historically be defined as "quasars." Their absolute magnitudes in the $B$ band (estimated roughly from their observed $R$-band flux) are $-18<M_{B}<-24$. Above the usual luminosity threshold defined for quasars $\left(M_{B}<-23\right)$, the unresolved AGN sample only includes 11 sources (or 23 sources if we include unresolved objects not selected in the radio, $\mathrm{X}$-ray, or IR). Therefore, our sample of optically unresolved AGNs would primarily be classified as luminous Seyfert galaxies. We note that it is not surprising that there are few luminous optical quasars in our sample at $0.25<z<0.8$. From the optical quasar luminosity function of Richards et al. (2005), we would expect only 18 objects with $M_{B}<-23$ in this redshift interval over the area of $7.3 \mathrm{deg}^{2}$ (after excluding bright stars), which probes a volume of $5 \times 10^{6} h^{-3} \mathrm{Mpc}^{3}$ at $0.25<z<0.8$.

\subsection{Overlap Between Samples}

One complication of selecting AGNs in different wavebands is that the samples overlap. Some AGNs are selected in more than one waveband, so the different AGN samples are not entirely independent. The various samples and their overlaps are given in Table 1, and a Venn diagram depicting the relative sizes and overlaps of the samples is shown in Figure 8. This figure shows the sample of sources that lie in the area covered by the radio, X-ray, and IRAC observations, to allow a fair comparison between the samples.

It is immediately apparent that there is very little overlap between the radio AGNs and those selected in the X-ray and



Figure 8. Venn diagram showing the relative number of AGNs with AGES redshifts at $0.25<z<0.8$, selected in the radio, X-ray, and IR (see also Table 1). The figure shows only those sources that lie within the regions covered by radio, X-ray, and IRAC observations (Figure 1). The overlapping areas between the samples correspond to the relative numbers selected in multiple wavebands, except for the region where all three overlap, which is slightly too small in the diagram. There is some overlap between the X-ray and IRAC samples, but little overlap between these and the radio AGNs.

(A color version of this figure is available in the online journal.)

Table 1

AGN Samples

\begin{tabular}{|c|c|c|c|c|c|c|}
\hline \multirow[t]{2}{*}{ Sample } & \multirow[t]{2}{*}{ Total $^{\mathrm{a}}$} & \multirow[t]{2}{*}{ Only ${ }^{b}$} & \multirow[t]{2}{*}{ Radio $^{c}$} & \multicolumn{2}{|c|}{ Also selected in } & \multirow[t]{2}{*}{$\mathrm{All}^{\mathrm{d}}$} \\
\hline & & & & X-Ray ${ }^{c}$ & IRAC $^{\mathrm{c}}$ & \\
\hline \multicolumn{7}{|c|}{ All AGNs } \\
\hline Radio & $122(122)$ & $103(103)$ & $\cdots$ & $6(6)$ & $6(6)$ & 7 \\
\hline X-ray & $362(296)$ & 238 (192) & $6(6)$ & $\ldots$ & $111(91)$ & 7 \\
\hline IRAC & 238 (199) & $114(95)$ & $6(6)$ & $111(91)$ & $\ldots$ & 7 \\
\hline \multicolumn{7}{|c|}{ Optically extended AGNs } \\
\hline Radio & $113(113)$ & $98(98)$ & $\ldots$ & $4(4)$ & $5(5)$ & 6 \\
\hline X-ray & $241(200)$ & $187(153)$ & $4(4)$ & $\cdots$ & $44(37)$ & 6 \\
\hline IRAC & $133(114)$ & $78(66)$ & $5(5)$ & $44(37)$ & $\cdots$ & 6 \\
\hline \multicolumn{7}{|c|}{ Optically unresolved AGNs } \\
\hline Radio & $9(9)$ & $5(5)$ & $\ldots$ & $2(2)$ & $1(1)$ & 1 \\
\hline X-ray & $121(96)$ & $51(39)$ & $2(2)$ & $\cdots$ & $67(54)$ & 1 \\
\hline IRAC & $105(85)$ & 36 (29) & $1(1)$ & $67(54)$ & $\ldots$ & 1 \\
\hline
\end{tabular}

Notes. Values in parentheses are for sources inside the region covered by radio, X-ray, and IRAC observations (Figure 1).

$a$ Total number of AGNs with AGES redshifts $0.25<z<0.8$, selected in waveband for that row, inside the main AGES field, away from bright stars.

${ }^{b}$ The number of AGNs selected in the waveband for that row only.

${ }^{c}$ The number of AGNs selected in the waveband for that row, and also in the band for that column only.

$d$ The number of AGNs selected in all three wavebands.

IR. This may provide an important clue as to the nature of the accretion in these systems (Section 9). However, there is significant overlap between the X-ray and IR samples. Roughly one-third of X-ray AGNs are in the IR sample, and half of the IR AGNs are detected in X-rays. These fractions are smaller (roughly $20 \%$ and $40 \%$, respectively) when we consider only optically extended AGNs. The sources that are selected as both X-ray and IRAC AGNs have relatively high luminosities; their median $L_{X}$ and $L_{4.5} \mu \mathrm{m}$ are $\simeq 50 \%$ higher than for AGNs selected in only the X-ray and IR bands, respectively. At some stages in the following analysis we study the properties of this 
joint X-ray and IR sample, but in most of the paper we consider the X-ray and IR AGN samples separately. This allows our results to be compared directly to other works that study X-ray or IR AGNs only, but it is important to keep in mind that the samples are not entirely independent.

It is also essential to stress that AGN selection is strongly affected by the flux limits of the observations in each waveband. In all three bands, our AGN selection criteria identify objects that are dominated by the AGN in that band. The luminosity cut in the radio, the X-ray flux limit, and the color criteria in the IR all select objects where the nucleus dominates over stellar processes at the observed wavelengths. However, as discussed in detail by Ho (2008), if observations go deep enough, then the majority of AGNs show signatures of nuclear activity in multiple bands, particularly in the X-ray and radio. Indeed, we show in Appendix B that many, if not most, of IR AGNs that are undetected in the shallow X-ray survey still have low-level Xray emission.

We also note that most X-ray AGNs are not selected by the IRAC color-color criteria, but in these objects the IRAC emission from the host galaxy may mask a relatively lowluminosity, red power-law-type SED from the AGN. Figure 7 shows that in the IRAC color-color diagram, many X-ray AGNs lie just below the Stern et al. (2005) selection region, which could be evidence for a low-level AGN contribution (Gorjian et al. 2008). It is possible that these objects contain relatively IR-faint AGNs that redden the colors of a quiescent galaxy and move it toward (but not all the way into) the AGN color-color selection region. However, IRAC color-color space below the Stern et al. (2005) region is also populated by quiescent galaxies with "green" colors similar to many Xray $\mathrm{AGN}$ hosts (Section 5), so the colors alone do not provide evidence for a weak AGN. In the future, a detailed study of the multiwavelength SEDs of these sources could put limits on the nuclear contribution to the IRAC flux.

\section{AGN HOST GALAXIES}

Links between AGN activity and the evolution of galaxies are reflected in the characteristics of the galaxies that host different classes of AGNs. As discussed in Section 3.3, a powerful diagnostic for understanding galaxy properties is their positions in optical color-magnitude space. In this section, we examine the optical colors and luminosities for AGNs with extended optical counterparts (corrected for nuclear contamination as described in Appendix A), and compare to the distribution for the full galaxy sample.

The color-magnitude diagrams for AGN hosts are shown in Figure 9(a). AGNs of all types have optical colors and luminosities that lie within, or close to, the parameter space occupied by normal galaxies, however, their distributions in color-magnitude space are markedly different from the total galaxy population, and vary significantly for AGNs selected in different wavebands.

Radio AGNs are concentrated on the luminous end of the red sequence, indicating that they reside in massive early-type galaxies, with only a few sources detected in the blue cloud (Figure 9, top panels). The mean and dispersion in ${ }^{0.1}(u-r)$ and $M_{0.1_{r}}$ are $\left(\langle C\rangle, \sigma_{C}\right)=(2.6,0.2)$ and $\left(\langle M\rangle, \sigma_{M}\right)=(-22.0,0.6)$, respectively. In contrast, X-ray AGNs are found throughout the galaxy color-magnitude space, with a disproportionate number found in the "green valley" (Figure 9, center panels). X-ray AGN hosts have $\left(\langle C\rangle, \sigma_{C}\right)=(2.2,0.4)$ and $\left(\langle M\rangle, \sigma_{M}\right)=$ $(-21.3,0.7)$. The fraction of AGES main sample galaxies containing X-ray AGNs is $4.6 \% \pm 0.7 \%$ in the "green valley" (having $A$ within \pm 0.2 mag of the division between red and blue galaxies). In contrast, the X-ray AGN fraction is $2.1 \% \pm$ $0.2 \%$ for galaxies with redder or bluer colors. As we discuss below, this enhanced X-ray AGN activity in green galaxies may reflect the connection between these objects and SMBH accretion.

IR-selected AGNs have a color distribution similar to that of X-ray AGNs, although they have a less pronounced excess in the "green valley," and have very few hosts on the red sequence (Figure 9, bottom panels), with $\left(\langle C\rangle, \sigma_{C}\right)=(2.1,0.4)$. The hosts of IR AGNs have smaller average optical luminosities than those X-ray AGNs, with $\left(\langle M\rangle, \sigma_{M}\right)=(-21.1,0.6)$. The sources that are selected as both X-ray and IRAC AGNs are found in a similar region of color-magnitude space to the full Xray and IR-selected samples, with $\left(\langle C\rangle, \sigma_{C}\right)=(2.2,0.4)$ and $\left(\langle M\rangle, \sigma_{M}\right)=(-21.2,0.6)$.

We conclude that IR and radio AGNs occupy almost entirely separate regions of the color-magnitude space, with radio AGNs in luminous red sequence galaxies, and IR AGNs in the less luminous blue and "green" hosts. X-ray AGNs are found in galaxies that are slightly redder and more luminous than the hosts of IR-selected AGNs.

\section{CORRELATION ANALYSIS}

Another important clue to the evolution of AGNs and their host galaxies comes from the large-scale environments in which AGNs are found. We derive the environments of AGNs and galaxies through a spatial two-point correlation analysis, which probes the clustering of sources on different comoving scales. We first calculate the autocorrelation of AGES main sample galaxies (and the red and blue samples separately), to determine the absolute linear bias of AGES galaxies relative to dark matter. Using the absolute bias, we then derive the characteristic masses of the dark matter halos which host galaxies of different types.

We next derive the cross-correlation of AGNs with AGES galaxies, to determine the relative bias between AGNs and galaxies, from which we derive the absolute bias and characteristic dark matter halo masses for the AGNs themselves. AGNgalaxy cross-correlation has been used since the first studies of quasar clustering (e.g., Bahcall et al. 1969) and has two main advantages, as discussed in Coil et al. (2007). First, it does not require a full understanding of the AGN selection function, which may be complicated, particularly for AGNs selected in multiple wavebands. Instead, we need to only know the selection function for the galaxies, which is well determined (Section 3). The second advantage is that AGN-galaxy cross-correlation has much greater statistical power than an AGN autocorrelation, owing to the large number of galaxies in the redshift survey (greater than 6000) compared to the number of AGNs (a few hundred).

The two-point correlation function $\xi(r)$ is defined as the probability above Poisson of finding a galaxy in a volume element $d V$ at a physical separation $r$ from another randomly chosen galaxy, such that

$$
d P=n[1+\xi(r)] d V
$$

where $n$ is the mean space density of the galaxies in the sample. To calculate the autocorrelation of AGES galaxies, we derive $\xi(r)$ using the Landy \& Szalay (1993) estimator:

$$
\xi(r)=\frac{1}{\mathrm{DD}}(\mathrm{DD}-2 \mathrm{DR}+\mathrm{RR}),
$$





Figure 9. (a) Optical colors and absolute magnitudes of AGNs with extended optical counterparts. Contours, black points, and dashed lines are as in Figure 4. Orange circles, green stars, and red squares show radio, X-ray, and IR AGNs, respectively. The arrows show the typical correction for nuclear contamination for different host galaxy colors, which range from 0 to $0.3 \mathrm{mag}$ (Appendix A). (b) Distribution in $A$ for AGNs selected in the three wavebands, compared to all AGES main sample galaxies at $0.25<z<0.8$ (scaled by $1 / 25$, thick gray line). The radio AGN color distribution peaks along the red sequence, while X-ray AGNs are found preferentially in the "green valley" between the red sequence and the blue cloud. The distribution of IR AGNs is similar to that of X-ray AGNs, although they are typically found in somewhat less luminous galaxies with very few on the red sequence, and show a less pronounced peak in the "green valley."

(A color version of this figure is available in the online journal.)

where $\mathrm{DD}, \mathrm{DR}$, and $\mathrm{RR}$ are the number of data-data, datarandom, and random-random galaxy pairs, respectively, at a separation $r$. We account for the sparse sampling of fainter galaxies by multiplying each pair by the product of the statistical weights of the observed (or random) galaxies. That is, when correlating galaxy sample 1 against galaxy sample 2, the weighted number of data-data pairs is

$$
\mathrm{DD}=\sum_{i \in \mathrm{D}_{1} \mathrm{D}_{2}} W_{1}^{i} W_{2}^{i},
$$

where $W_{1}^{i}$ and $W_{2}^{i}$ for each pair are the statistical weights of the galaxies from samples 1 and 2, respectively. By including these weights, we ensure that brightest galaxies do not overly dominate the correlation function. We correspondingly include the random galaxy weights (Section 3.4) in calculating DR and $\mathrm{RR}$. The random galaxy weights are assigned so that their total weight equals that of the AGES galaxies, so that DD, DR, and RR may be compared directly.

Following Coil et al. (2007), for the cross-correlation between AGNs and galaxies we use the simple estimator

$$
\xi(r)=\frac{D_{1} D_{2}}{D_{1} R_{2}}-1,
$$

where $\mathrm{D}_{1} \mathrm{D}_{2}$ is the number of AGN-galaxy pairs and $D_{1} R_{2}$ is the number of AGN-random pairs. Because the selection function of the AGN samples is not as well defined as that for the AGES main sample galaxies, we do not assign statistical weights to the AGNs. Therefore, we use only the galaxy weights in calculating the weighted number of pairs, such that

$$
\mathrm{D}_{1} \mathrm{D}_{2}=\sum_{i \in \mathrm{D}_{1} \mathrm{D}_{2}} W_{2}^{i},
$$

and correspondingly for $\mathrm{D}_{1} \mathrm{R}_{2}$.

In the range of separations $1 \lesssim r \lesssim 10 h^{-1} \mathrm{Mpc}, \xi(r)$ for galaxies is roughly observed to be a power law, $\xi(r)=\left(r / r_{0}\right)^{-\gamma}$, although recent work has shown evidence for separate terms in the correlation owing to dark matter halos that host single galaxies and those that host pairs of galaxies (e.g., Zehavi et al. 2004; Zheng et al. 2007, 2009; Coil et al. 2008; Brown et al. 2008). For simplicity, and in light of the uncertainties in the 
AGN-galaxy correlations, we will restrict our fits to powerlaw models. Throughout the paper $r_{0}$ is given in comoving coordinates.

\subsection{The Projected Correlation Function}

With a redshift survey, we cannot directly measure $\xi(r)$ in physical space, because peculiar motions of galaxies distort the line-of-sight distances measured from redshifts. To account for these redshift-space distortions, we instead measure the correlation function $\xi\left(r_{p}, \pi\right)$, where $r_{p}$ and $\pi$ are the projected comoving separations between galaxies in the directions perpendicular and parallel, respectively, to the mean line of sight between the two galaxies.

Redshift space distortions only affect the correlation function along the line of sight, so we integrate $\xi\left(r_{p}, \pi\right)$ along the $\pi$ direction to obtain the statistic,

$$
w_{p}\left(r_{p}\right)=2 \int_{0}^{\pi_{\max }} \xi\left(r_{p}, \pi\right) d \pi
$$

which is independent of redshift space distortions (following Davis \& Peebles 1983). This estimator has been used to measure correlations in a number of surveys, for example, SDSS (Zehavi et al. 2005; Li et al. 2006), 2SLAQ (Wake et al. 2008), DEEP2 (Coil et al. 2007, 2008), and GOODS (Gilli et al. 2007). If $\pi_{\max }=\infty$, then we average over all line-of-sight peculiar velocities, and $w_{p}\left(r_{p}\right)$ can be directly related to $\xi(r)$ (for a power-law parameterization) by

$$
w_{p}\left(r_{p}\right)=r_{p}\left(\frac{r_{0}}{r_{p}}\right)^{\gamma} \frac{\Gamma(1 / 2) \Gamma[(\gamma-1) / 2]}{\Gamma(\gamma / 2)} .
$$

In practice, we truncate the integral at a finite $\pi_{\max }$, to reduce the noise from integrating over weakly correlated objects at large $\pi$. Here, we use $\pi_{\max }=25 h^{-1}$ Mpc. Because of the finite $\pi_{\max }$, in order to recover $r_{0}$ and $\gamma$ exactly we must account for redshift-space distortions due to peculiar motions along the line of sight. This requires an accounting for coherent infall of galaxies on large scales, as well as the random velocities of galaxies on small scales ("fingers of God").

We account for these redshift-space distortions using the method of Coil et al. (2007, 2008). Coherent infall depends on the parameter $\beta \approx \Omega_{\mathrm{m}}^{0.6} / b$ (Kaiser 1987), where $\Omega_{\mathrm{m}}$ is evaluated at the mean redshift of the sample, and $b$ is the absolute bias relative to dark matter. For $z=0.5$ (the mean redshift for the AGNs), $\Omega_{\mathrm{m}}=0.59$. We take $b=1.23$, the absolute bias of AGES galaxies at $0.25<z<0.8$ (see Section 6.3 and Section 7), so that $\beta=0.59$. For the pairwise random velocities of galaxies, we take $\sigma_{12}=500 \mathrm{~km} \mathrm{~s}^{-1}$, which is characteristic of galaxies with luminosities typical of the AGES sample (e.g., Hawkins et al. 2003; Coil et al. 2008). For reasonable variations in $\beta( \pm 0.1)$ or $\sigma_{12}\left( \pm 400 \mathrm{~km} \mathrm{~s}^{-1}\right)$, the resulting $w_{p}\left(r_{p}\right)$ changes by at most a few percent.

We then derive $\xi\left(r_{p}, \pi\right)$ in redshift space as a function of $r_{0}$ and $\gamma$, including coherent infall and random velocities as described in Section 4.1 of Hawkins et al. (2003). We numerically integrate $\xi\left(r_{p}, \pi\right)$ to $\pi_{\max }=25 h^{-1} \mathrm{Mpc}$ to determine a model $w_{p}\left(r_{p}\right)$ which we fit to the data. For a given $r_{0}$ and $\gamma$, this $w_{p}\left(r_{p}\right)$ differs from that given in Equation (9) by less than $4 \%$ for $r_{p}<10 \mathrm{~h}^{-1} \mathrm{Mpc}$. Accordingly, the best-fit $r_{0}$ and $\gamma$ differ from those using Equation (9) by less than a few percent.

\subsection{Calculating Relative Bias}

For each subset of galaxies and AGNs, we also calculate the relative bias $b_{\text {rel }}$ compared to the autocorrelation of all AGES main sample galaxies. The relative bias for the red galaxy sample is given by

$$
b_{\mathrm{rel}}^{2}=\frac{w_{p}\left(r_{p}\right)[\mathrm{red}-\mathrm{red}]}{w_{p}\left(r_{p}\right)[\mathrm{gal}-\mathrm{gal}]},
$$

and accordingly for blue galaxies. For AGNs, since we calculate the AGN-galaxy cross-correlation, the relative bias is

$$
b_{\mathrm{rel}}=\frac{w_{p}\left(r_{p}\right)[\mathrm{AGN}-\mathrm{gal}]}{w_{p}\left(r_{p}\right)[\mathrm{gal}-\mathrm{gal}]} .
$$

In each case we derive $b_{\text {rel }}$ as a function of $r_{p}$, and calculate the mean biases averaged over scales of $1-10 h^{-1} \mathrm{Mpc}$ and $0.3-$ $1 h^{-1}$ Mpc.

The galaxy autocorrelation varies with redshift, owing to the evolution of large-scale structure, and because the use of a fluxlimited sample means we selected more luminous galaxies at higher $z$. This can affect bias measurements, since the redshift distribution of the AGN tends to peak at higher $z$ than that for the galaxies. To account for this, for each AGN sample we assign weights to the AGES galaxies and the random galaxy sample, so that their (weighted) redshift distribution is the same as that of the AGNs in bins of size $\Delta z=0.1$. Including these redshift weights, we rederive the autocorrelation of the AGES main AGES sample, correspondingly weighting the random galaxy sample, and multiplying each galaxy-galaxy, galaxy-random, or random-random pair by these additional weights. We then use this redshift-matched autocorrelation function when calculating the bias of AGNs relative to galaxies.

Furthermore, for AGNs with extended optical counterparts, it is interesting to compare the AGN-galaxy cross-correlation with that measured for galaxies with similar color classification, redshift, and absolute magnitude. To create this comparison sample, we assign additional weights to the AGES main sample galaxies, so that their (weighted) distribution in color, absolute magnitude, and redshift is the same as that for the AGN hosts (in bins of $0.25 \mathrm{mag}, 0.25 \mathrm{mag}$, and 0.1 , respectively). We then calculate the cross-correlation $w_{p}\left(r_{p}\right)$ between this comparison sample and all AGES galaxies (again multiplying each galaxygalaxy or galaxy-random pair by these additional weight) and derive the bias (on scales $1-10$ and $0.3-1 h^{-1} \mathrm{Mpc}$ ) for the AGNs relative to this cross-correlation function.

\subsection{Absolute Bias and Dark Matter Halo Mass}

As discussed in Section 1, the mass of the parent dark matter halo may be a key parameter in determining the evolution of galaxies and their central black holes. We therefore use our clustering results to estimate $M_{\text {halo }}$ for our different subsets of galaxies and AGNs. The masses of dark matter halos are reflected in their absolute bias relative to the dark matter distribution. To determine absolute bias (following Coil et al. 2008) we first calculate the two-point autocorrelation of dark matter as a function of redshift, using the code of Smith et al. (2003), and assuming our standard cosmology, with $\sigma_{8}=0.9$ and the slope of the initial fluctuation power spectrum, $\Gamma=\Omega_{m} h=0.21$. We derive $w_{p}\left(r_{p}\right)$ as a function of redshift for the dark matter, integrating to $\pi=25 h^{-1} \mathrm{Mpc}$ as for the data. We assume an uncertainty in the model dark matter correlation function of $5 \%$ at each scale. 
For each subset of AGNs, we next divide the projected autocorrelation function for galaxies that are matched in redshift (Section 6.2) by the $w_{p}\left(r_{p}\right)$ for the dark matter, averaged over the redshift distribution of the sample. The mean ratio between these two correlation functions on scales $1<r_{p}<10 h^{-1}$ Mpc gives $b_{\text {gal }}^{2}$, where $b_{\text {gal }}$ is the absolute linear bias of AGES galaxies with given redshift distribution. The absolute bias of the AGN sample is then $b_{\mathrm{abs}}=b_{\mathrm{gal}} b_{\text {rel }}$, where $b_{\text {rel }}$ is the bias relative to AGES galaxies, determined by the cross-correlation (Section 6.2).

Finally, we use $b_{\mathrm{abs}}$ to derive the characteristic mass of the dark matter halos hosting each subset of galaxies or AGNs. The halo masses presented in this paper represent the virial mass in the sense of a top-hat spherical collapse model (see, e.g., Peebles 1993, and references therein). To obtain halo mass, we first convert $b_{\text {abs }}$ to the quantity $v=\delta_{c} / \sigma(M)$, where $\sigma(M)$ is the linear theory rms mass fluctuation in spheres of radius $r=(3 M / 4 \pi \bar{\rho})^{1 / 3}\left(\bar{\rho}\right.$ is the background density) and $\delta_{c} \approx 1.69$ is the critical overdensity required for collapse. We convert $b_{\mathrm{abs}}$ to $v$ using Equation (8) of Sheth et al. (2001), and use $v$ to derive $M_{\text {halo }}$ following Appendix A of van den Bosch (2002). If we use a different relation between $b_{\mathrm{abs}}$ and $v$ (Tinker et al. 2005), we obtain estimates for $M_{\text {halo }}$ that are similar, although slightly larger by $0.1-0.2$ dex.

We note that to estimate $M_{\text {halo }}$, we have averaged the absolute bias on scales 1-10 $h^{-1} \mathrm{Mpc}$. On these scales, the dark matter and galaxy correlation functions can have somewhat different shapes, so that the relative bias is a function of scale (e.g., Cresswell \& Percival 2009). To verify our method for estimating $M_{\text {halo }}$ using the 1-10 $h^{-1}$ Mpc bias, we compared the $b_{\text {abs }}-$ $M_{\text {halo }}$ relation of Sheth et al. (2001) to a relation derived from the dark matter simulations of Padmanabhan et al. (2009). Using the simulation results for $z \approx 0.5$, we divided the $w_{p}\left(r_{p}\right)$ for halos of different masses (evaluated between 1 and $\left.10 h^{-1} \mathrm{Mpc}\right)$ to the $w_{p}\left(r_{p}\right)$ from the dark matter model of Smith et al. (2003; calculated using the same cosmology assumed by Padmanabhan et al. 2009). For a given $1-10 h^{-1} \mathrm{Mpc}$ bias, the corresponding $M_{\text {halo }}$ from the simulations matches the prediction of Sheth et al. (2001) to better than 0.08 dex. This confirms that measuring bias from 1-10 $h^{-1} \mathrm{Mpc}$ scales allows sufficiently accurate estimates of $M_{\text {halo }}$. Finally, we check that our estimates of $M_{\text {halo }}$ are relatively insensitive to our choice of $\sigma_{8}$. Decreasing $\sigma_{8}$ results in weaker dark matter clustering and so larger $b_{\text {abs }}$ for galaxies with a given observed $w_{p}\left(r_{p}\right)$. However, decreasing $\sigma_{8}$ also reduces the $M_{\text {halo }}$ corresponding to a given $b_{\text {abs }}$, so there is little change in our estimates of $M_{\text {halo }}$. If we change $\sigma_{8}$ from 0.9 to 0.8 , our $M_{\text {halo }}$ estimates for AGES galaxies and AGNs vary by less than \pm 0.1 dex.

In addition to top-hat virial mass, another commonly used mass estimator is $M_{200}$, which is equal to the mass contained within the radius where the overdensity is 200 times the critical density. For our chosen cosmology, $M_{200}$ is slightly smaller than the top-hat virial mass, by $\approx 20 \%$ (White 2001).

\subsection{Uncertainties}

We determine uncertainties in $w_{p}\left(r_{p}\right)$ directly from the data, in two ways. For the galaxy autocorrelations, errors are derived from jackknife resampling, which generally approximates the variance in $w_{p}\left(r_{p}\right)$ derived from simulated galaxy catalogs (e.g., Zehavi et al. 2005; Coil et al. 2008). We divide the sample into five separate regions (large enough to sample all the appropriate scales) and recalculate $w_{p}\left(r_{p}\right)$ excluding each region in turn.
The uncertainty in $w_{p}\left(r_{p}\right)$ is taken to be the variance between the jackknife samples. However, when calculating $w_{p}\left(r_{p}\right)$ for the AGN samples, jackknife resampling involves only a small number of sources, so the uncertainties on different scales vary significantly. Therefore, for the optically extended AGNs we instead create a set of 100 random comparison galaxy samples matched in color, absolute magnitude, and redshift (as described in Section 6.2), but containing only one galaxy corresponding to each AGN. We calculate $w_{p}\left(r_{p}\right)$ for each of the 100 samples, and take the variance to be the uncertainty in $w_{p}\left(r_{p}\right)$. These uncertainties are typically $\sim 2$ times the corresponding jackknife errors. We similarly calculate the variance in the bias (relative to all galaxies, and to the matched sample) and the best-fit power law parameters, to account for covariance in different bins of $r_{p}$. For optically unresolved AGNs, we cannot create a matched sample of galaxies, so we simply repeat the calculation for the optically extended sources of each type (radio, X-ray, and IR), but vary the sample size to match the corresponding number of unresolved AGNs and take as the uncertainties the variance in $w_{p}$ and bias between the 100 samples.

We perform power-law $\chi^{2}$ fits to $w_{p}$ (using the uncertainties derived from the above resampling) over the range $0.3<$ $r_{p}<10 h^{-1} \mathrm{Mpc}$, from which we obtain the best-fit $r_{0}$ and $\gamma$. We estimate the uncertainties in $r_{0}$ and $\gamma$ by the variance in the best-fit parameters between the jackknife samples (for the galaxy autocorrelation) or the 100 comparison samples (for the AGN-galaxy cross-correlation), to account for covariance between bins of $r_{p}$. We note that our jackknife resampling appears to underestimate the uncertainties in the fit parameters by about $3 \%$ of the values of $r_{0}$ and $\gamma$, compared to variance in simulated galaxy catalogs with comparable source densities and number of objects (Coil et al. 2008). We therefore include a systematic uncertainty of $\pm 3 \%$ on the best-fit parameters and on the absolute bias. This gives total uncertainties for the galaxy autocorrelations that are similar to those derived from simulations, but has a negligible effect on the (much larger) errors for the cross-correlation of AGNs and galaxies.

\section{CORRELATION RESULTS}

In this section, we discuss the results of the correlation analysis and the characteristic dark matter halo masses for galaxies and AGNs.

\subsection{Galaxy Autocorrelation}

As a first exercise, we measure the autocorrelation of the main AGES galaxy sample, as well as for the red and blue galaxy samples separately. The resulting $w_{p}\left(r_{p}\right)$ for the galaxy autocorrelations are shown in Figure 10, and the parameters of power-law fits to the data (including the reduced $\chi^{2}$ ) are given in Table 2. The range of absolute magnitudes for the red and blue galaxy samples are $-19 \lesssim M_{0.1} r-5 \log h \lesssim-23$ and $-19 \lesssim$ $M_{0.1}-5 \log h \lesssim-22.5$, respectively. The autocorrelation of the main galaxy sample is best fit by $r_{0}=4.6 \pm 0.2 h^{-1} \mathrm{Mpc}$ and $\gamma=1.8 \pm 0.1$. This is broadly consistent with previous results (see, e.g., Table 4 in Brown et al. 2003; Table 1 of Coil et al. 2008; Brown et al. 2008, and references therein). The corresponding absolute bias indicates that typical AGES galaxies reside in dark matter halos with $M_{\text {halo }} \simeq 10^{13} h^{-1} M_{\odot}$.

Dividing the galaxy sample into red and blue subsets, we find that red galaxies show a significantly stronger autocorrelation and a steeper slope than the blue galaxies, reflecting the well established relationship between color and clustering. The 


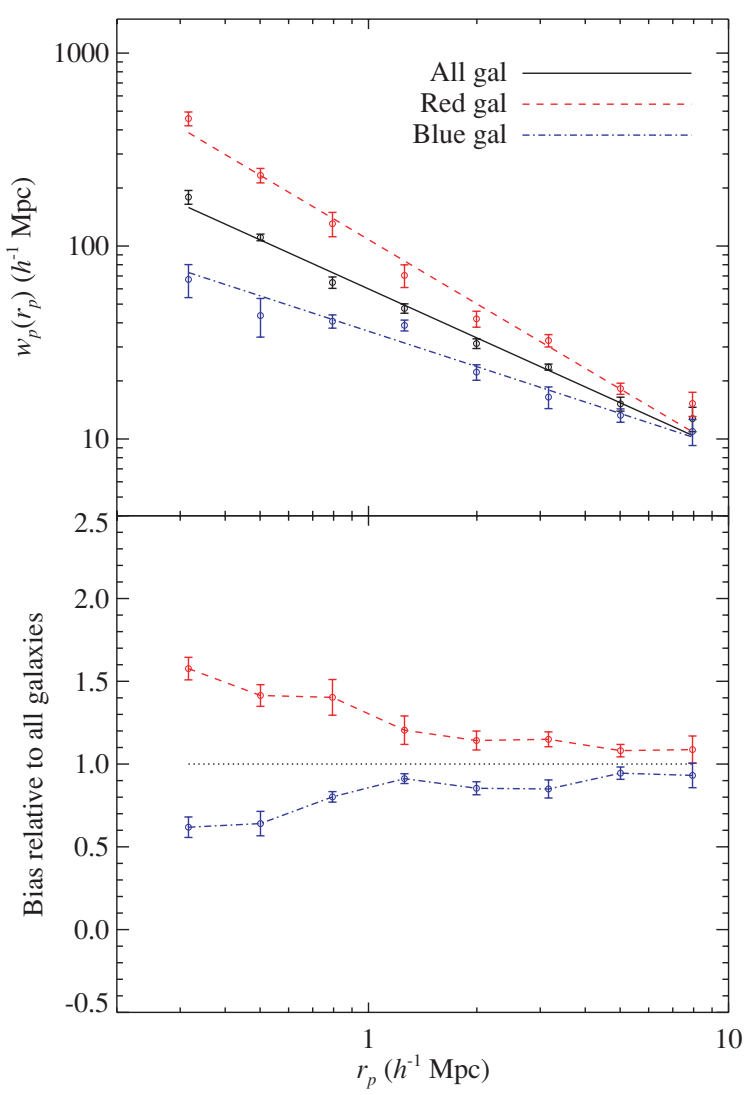

Figure 10. Top panel shows galaxy autocorrelations in the AGES galaxy sample. The points show the projected autocorrelation function in the redshift interval $0.25<z<0.8$ for all galaxies in the main AGES sample (black), red AGES galaxies (red), and blue AGES galaxies (blue). Uncertainties are taken from jackknife resampling, and lines show power-law fits to the data. The bottom panel shows bias relative to the main galaxy sample, defined as the square root of the ratio between $w_{p}\left(r_{p}\right)$ for the autocorrelation of red (or blue) galaxies and $w_{p}\left(r_{p}\right)$ for the autocorrelation of all AGES galaxies. Real-space fit parameters and average bias values are given in Table 2.

(A color version of this figure is available in the online journal.)

difference in clustering between red and blue galaxies is not simply a luminosity effect. If we select random samples of red and blue galaxies that are uniformly distributed in bins of 0.25 mag between $-19>M_{0.1}>-21.25$, the relative bias between red and blue galaxies changes by only $2 \%$. We note that the $w_{p}\left(r_{p}\right)$ is not exactly a power law, especially for the red galaxy sample. The apparent upturn of $w_{p}\left(r_{p}\right)$ on scales less than $1 h^{-1}$ Mpc is indicative of an additional "one-halo" term in the correlation function, corresponding to dark matter halos that contain multiple galaxies (e.g., Zehavi et al. 2004; Zheng et al. 2007, 2009; Coil et al. 2008; Brown et al. 2008).

As a check, we also perform the galaxy autocorrelation using an alternative clustering measure, the $\omega$ statistic of Padmanabhan et al. (2007). This statistic has the advantage of being less sensitive than $w_{p}\left(r_{p}\right)$ to large-scale structures and the effects of truncating the line-of-sight integral at $\pi_{\max }$. In addition, it is possible to evaluate $\omega(R)$ by converting the integrals to Riemann sums, and so it does not require binning the data. Using the $(2,2)$ filter preferred in Padmanabhan et al. (2007), and evaluating their Equation (29), we derive $\omega(R)$ and calculate jackknife uncertainties as described above. We perform a power-law fit to $\omega$ and derive $r_{0}$ and $\gamma$ using Equation (24) of Padmanabhan et al. (2007). The best-fit values are $r_{0}=4.8$ and $\gamma=2.0$, within $5 \%$ and $7 \%$, respectively $(\sim 1 \sigma)$ of the values derived from the $w_{p}\left(r_{p}\right)$ fit. We note, that since



Figure 11. Projected cross-correlations between AGNs and all AGES galaxies. The top panel shows the projected cross-correlation between radio AGNs (orange circles), X-ray AGNs (green stars), and IR AGNs (red squares), and all AGES galaxies in the redshift interval $0.25<z<0.8$. For comparison, the dotted lines show galaxy autocorrelations for the main AGES galaxy samples, weighted to have similar weighted redshift distributions to the combined sample of AGNs. The bottom panel shows bias relative to AGES galaxies, defined as the ratio between $w_{p}\left(r_{p}\right)$ for the AGN-galaxy cross-correlation and $w_{p}\left(r_{p}\right)$ for the galaxy autocorrelation.

(A color version of this figure is available in the online journal.)

$\omega(R)$ involves an integral over $r_{p}$ as well as $\pi$, the bins of $\omega(R)$ are not independent. We therefore use $w_{p}\left(r_{p}\right)$ to estimate the AGN-galaxy cross-correlation and relative bias as a function of scale.

\subsection{AGN-Galaxy Cross-Correlation}

We next measure the cross-correlation of AGNs with AGES galaxies, which gives us the bias of AGNs relative to galaxies, from which we derive the absolute bias and $M_{\text {halo }}$ for the AGNs. We first perform the AGN-galaxy cross-correlations for all AGNs (including those with extended or unresolved counterparts). The $w_{p}\left(r_{p}\right)$ values are shown in Figure 11, and the parameters of power-law fits to the correlation functions are given in Table 2. We further divide the AGN samples into those with extended or unresolved optical counterparts (Figures 12 and 13). For optically extended sources, we compare the clustering of AGN hosts to a control sample of normal galaxies that are appropriately weighted to have similar colors, absolute magnitudes, and redshifts as the AGN hosts (Section 6.2). Here, we discuss the results for the different classes of AGNs, which show significant differences in clustering.

\subsubsection{Radio AGNs}

The radio AGNs are most strongly clustered, with significant bias relative to normal galaxies indicating that they reside 
Table 2

Correlation Results ${ }^{\mathrm{a}}$

\begin{tabular}{|c|c|c|c|c|c|c|c|c|c|c|c|}
\hline \multirow[t]{3}{*}{ Subset } & \multirow[t]{3}{*}{$N_{\text {src }}$} & \multirow[t]{3}{*}{$\langle z\rangle^{\mathrm{b}}$} & \multirow[t]{3}{*}{$r_{0}$} & \multirow[t]{3}{*}{$\gamma$} & \multirow[t]{3}{*}{$\chi_{v}^{2}$} & \multicolumn{4}{|c|}{ Relative Bias } & \multirow{3}{*}{$\begin{array}{c}\text { Absolute Bias } \\
1<r_{\mathrm{p}}<10\end{array}$} & \multirow{3}{*}{$\begin{array}{c}\text { Characteristic } \\
\text { Halo Mass } \\
\left(\log h^{-1} M_{\odot}\right)\end{array}$} \\
\hline & & & & & & \multicolumn{2}{|c|}{ vs. All Galaxies ${ }^{c}$} & \multicolumn{2}{|c|}{ vs. Matched Sample ${ }^{\mathrm{d}}$} & & \\
\hline & & & & & & $1<r_{\mathrm{p}}<10$ & $0.3<r_{\mathrm{p}}<1$ & $1<r_{\mathrm{p}}<10$ & $0.3<r_{\mathrm{p}}<1$ & & \\
\hline \multicolumn{12}{|c|}{ Galaxies } \\
\hline All & 6262 & 0.39 & $4.6 \pm 0.2$ & $1.8 \pm 0.1$ & 1.9 & 1 & 1 & $\cdots$ & $\cdots$ & $1.19 \pm 0.04$ & $12.6_{-0.1}^{+0.1}$ \\
\hline Red & 3146 & 0.41 & $5.3 \pm 0.2$ & $2.1 \pm 0.1$ & 2.2 & $1.13 \pm 0.05$ & $1.45 \pm 0.06$ & $\cdots$ & $\cdots$ & $1.37 \pm 0.08$ & $13.0_{-0.1}^{+0.1}$ \\
\hline Blue & 3116 & 0.38 & $3.8 \pm 0.2$ & $1.6 \pm 0.1$ & 1.8 & $0.89 \pm 0.08$ & $0.71 \pm 0.08$ & $\cdots$ & $\cdots$ & $1.04 \pm 0.09$ & $12.2_{-0.4}^{+0.1}$ \\
\hline \multicolumn{12}{|c|}{ All AGNs } \\
\hline Radio & 122 & 0.57 & $6.3 \pm 0.6$ & $1.8 \pm 0.2$ & 3.5 & $1.51 \pm 0.14$ & $1.70 \pm 0.25$ & $\cdots$ & $\cdots$ & $2.03 \pm 0.20$ & $13.5_{-0.2}^{+0.1}$ \\
\hline X-ray & 362 & 0.51 & $4.7 \pm 0.3$ & $1.6 \pm 0.1$ & 0.8 & $1.08 \pm 0.12$ & $0.66 \pm 0.15$ & $\cdots$ & $\cdots$ & $1.40 \pm 0.16$ & $12.9_{-0.3}^{+0.2}$ \\
\hline IRAC & 238 & 0.48 & $3.7 \pm 0.4$ & $1.5 \pm 0.1$ & 0.4 & $0.75 \pm 0.14$ & $0.46 \pm 0.18$ & $\cdots$ & $\cdots$ & $0.95 \pm 0.18$ & $11.7_{-1.5}^{+0.6}$ \\
\hline$X$-ray and IRAC ${ }^{f}$ & 118 & 0.53 & $3.9 \pm 0.7$ & $1.6 \pm 0.3$ & 0.7 & $0.77 \pm 0.21$ & $0.51 \pm 0.33$ & $\cdots$ & $\cdots$ & $0.99 \pm 0.27$ & $11.9_{-5.9}^{+0.7}$ \\
\hline \multicolumn{12}{|c|}{ AGNs (Galaxies) } \\
\hline Radio & 113 & 0.55 & $6.1 \pm 0.5$ & $1.8 \pm 0.2$ & 3.4 & $1.46 \pm 0.15$ & $1.68 \pm 0.28$ & $1.03 \pm 0.11$ & $0.91 \pm 0.15$ & $1.95 \pm 0.21$ & $13.5_{-0.2}^{+0.2}$ \\
\hline X-ray & 241 & 0.48 & $4.6 \pm 0.4$ & $1.6 \pm 0.1$ & 0.3 & $1.05 \pm 0.12$ & $0.65 \pm 0.22$ & $1.01 \pm 0.11$ & $0.66 \pm 0.23$ & $1.34 \pm 0.15$ & $12.8_{-0.3}^{+0.2}$ \\
\hline IRAC & 133 & 0.42 & $3.1 \pm 0.7$ & $1.2 \pm 0.3$ & 0.3 & $0.73 \pm 0.18$ & $0.26 \pm 0.33$ & $0.70 \pm 0.17$ & $0.34 \pm 0.36$ & $0.90 \pm 0.20$ & $11.6_{-5.6}^{+0.8}$ \\
\hline \multicolumn{12}{|c|}{ AGNs (Unresolved) $)^{\mathrm{g}}$} \\
\hline X-ray & 121 & 0.57 & $5.0 \pm 0.7$ & $1.7 \pm 0.2$ & 1.3 & $1.14 \pm 0.17$ & $0.69 \pm 0.25$ & $\cdots$ & $\cdots$ & $1.52 \pm 0.23$ & $13.0_{-0.4}^{+0.3}$ \\
\hline IRAC & 105 & 0.55 & $4.1 \pm 0.7$ & $2.0 \pm 0.2$ & 2.2 & $0.70 \pm 0.18$ & $0.81 \pm 0.27$ & $\cdots$ & $\cdots$ & $0.93 \pm 0.24$ & $11.6_{-5.6}^{+0.8}$ \\
\hline
\end{tabular}

Notes. All units for $r_{\mathrm{p}}$ and $r_{0}$ are in $h^{-1} \mathrm{Mpc}$ (comoving).

${ }^{a}$ Results in the top three rows are for the autocorrelation of all AGES main sample galaxies, red AGES galaxies, and blue AGES galaxies, derived using the Landy \& Szalay (1993) estimator (Equation (4)). Values in the other rows are for the cross-correlation of the given AGN sample with all AGES main sample galaxies, derived using the estimator given by Equation (6).

b Median redshift for the sources in the sample.

${ }^{c}$ Bias relative to AGES main sample galaxies with matched redshift distributions (Section 6.2), averaged over the scales is shown.

${ }^{\mathrm{d}}$ Bias relative to AGES main sample galaxies with matched distributions in color, absolute magnitude, magand redshift (Section 6.2), averaged over the scales is shown.

e Virial mass in the sense of a top-hat collapse model, assuming a cosmology with $\Omega_{m}=0.3, \Omega_{\Lambda}=0.7$, and $\sigma_{8}=0.9$.

f AGNs selected both in the X-rays and IR.

g Optically unresolved radio AGNs are not included in the clustering analysis, owing to the small sample size and the corresponding large uncertainties.

in massive dark matter halos $\left(M_{\text {halo }} \sim 3 \times 10^{13} h^{-1} M_{\odot}\right)$ characteristic of large galaxy groups or small clusters. There is an increase in bias on scales less than $0.5 h^{-1} \mathrm{Mpc}$, similar to that seen for red AGES galaxies. This suggests that on small scales, the cross-correlation of radio AGNs with galaxies is dominated by pairs of objects in the same dark matter halo, which is to be expected if these sources reside in massive systems (e.g., Zehavi et al. 2004; Zheng et al. 2007, 2009; Coil et al. 2008; Brown et al. 2008).

Interestingly, compared to a control sample of AGES galaxies matched in color, $M_{0.1}$, and $z$, the radio AGNs show no significant difference in clustering on all scales from 0.3 to $10 \mathrm{~h}^{-1} \mathrm{Mpc}$. This is apparently in contrast to recent studies that found that radio AGNs are more strongly clustered (and have correspondingly larger halo masses) compared to matched control samples of galaxies. For example, Mandelbaum et al. (2009) selected radio AGNs from SDSS and found that their dark matter halos are roughly twice as massive as those for galaxies matched in redshift, stellar mass, and stellar velocity dispersion. Wake et al. (2008) found a similar effect for more luminous radio AGNs at $0.4<z<0.7$ taken from the 2SLAQ Luminous Red Galaxy survey, with the radio sample inhabiting $\sim 2$ times larger halos than the control galaxies matched in luminosity and color.

We note that the differences between these studies and our own results are only marginally significant; if the bias of the Boötes radio AGNs were increased by $1.5 \sigma$, this would correspond to a factor of 2 larger halo mass relative to the control galaxies, and would match the results of Mandelbaum et al. and Wake et al. Nonetheless, it may be worth noting that Mandelbaum et al. (2009) studied a subsample of high stellar- mass objects that corresponds closely to the range of $P_{1.4 \mathrm{GHz}}$ and host galaxy mass probed in Boötes (assuming mass-to-light ratios from Kauffmann et al. 2003b), and found that for these objects, the difference in clustering between the radio AGNs and the control sample is small.

These results may hint at an interesting point regarding the fueling of radio AGNs. In particular, the importance of environment in triggering radio activity may depend on the radio luminosity relative to the galaxy's stellar mass. Compared to the Boötes radio AGNs, the full Mandelbaum et al. (2009) sample extends to lower stellar masses, while the Wake et al. AGNs have similar host galaxy absolute magnitudes but higher radio luminosities (their median $P_{1.4 \mathrm{GHz}}$ is $\sim 3$ times higher than that in our sample; Sadler et al. 2007). Therefore, both samples probe sources with a higher typical ratio of radio power to stellar mass than the Boötes sample (or in the Mandelbaum et al. high-mass sample), for which we see little clustering difference between radio AGNs and control galaxies.

This suggests that for galaxies with low radio luminosities (relative to their stellar masses), environment may have little effect on the fueling of radio activity. This is certainly true in the limit of very low luminosity radio AGNs, which are found in virtually every massive galaxy (Sadler et al. 1989). However, the fueling of AGNs with higher radio power relative to galaxy mass, which are less common than the low-power sources (Best et al. 2005), may be more strongly dependent on the mass of the host dark matter halo. In the future, a larger study that examines radio AGN clustering over a wide range of radio luminosities and environments will allow us to better understand how radio activity is triggered in halos of different masses. 




Figure 12. Same as Figure 11, for only those sources with extended optical counterparts (Section 5). Real-space fit parameters and average bias values are given in Table 2

(A color version of this figure is available in the online journal.)

\subsubsection{X-ray AGNs}

For the X-ray AGNs, the clustering is consistent with that of typical AGES galaxies on scales of $1-10 h^{-1} \mathrm{Mpc}$, indicating that they reside in halos with $M_{\text {halo }} \sim 10^{13} h^{-1} M_{\odot}$, characteristic of poor to moderate-sized galaxy groups. There is little difference in the clustering between the optically unresolved and optically extended X-ray AGN samples. This suggests that obscured or optically faint AGNs show similar clustering to their optically bright counterparts. This is analogous to the well established results for optical quasars, for which clustering is only weakly (if at all) dependent on optical luminosity (e.g., Adelberger \& Steidel 2005; Porciani \& Norberg 2006; Myers et al. 2007; da Ângela et al. 2008), although unlike in optical quasar samples, some of our X-ray AGNs are likely optically faint because of obscuration, rather than low intrinsic luminosity. Relative to a matched sample of normal galaxies, the optically extended X-ray AGNs show no significant bias.

However, on scales less than $1 h^{-1} \mathrm{Mpc}$, the clustering of Xray AGNs differs significantly from that of normal galaxies. The slope of the AGN-galaxy cross-correlation function for X-ray AGNs is significantly flatter than the autocorrelation function for normal AGES galaxies, such that the X-ray AGNs are significantly antibiased on small scales $\left(0.3-1 h^{-1} \mathrm{Mpc}\right)$. This antibias on small scales is found for all X-ray AGNs relative to all AGES galaxies, and also for optically extended X-ray AGNs, relative to the control sample of normal galaxies with matched optical properties.



Figure 13. Same as Figure 11, for only those sources with unresolved optical counterparts (Section 5). There are only nine radio-selected, optically unresolved AGNs, so the corresponding $w_{p}\left(r_{p}\right)$ values have large uncertainties and are not included here. Real-space fit parameters and average bias values are given in Table 2 .

(A color version of this figure is available in the online journal.)

A similar result was recently obtained by $\mathrm{Li}$ et al. (2006) for optically selected narrow-line AGN in SDSS. Specifically, Li et al. (2006) found that on scales greater than $1 h^{-1} \mathrm{Mpc}$, the cross-correlation between optical AGNs and galaxies is similar to a control sample of normal galaxies, but that the AGN-galaxy clustering is weaker on scales less than $1 h^{-1} \mathrm{Mpc}$. Comparing to mock galaxy catalogs taken from cosmological simulations, Li et al. (2006) showed that the small-scale antibias can naturally be explained if the AGNs are preferentially located in central galaxies within their dark matter halos, which would serve to suppress the "one-halo" term corresponding to strong clustering on small scales. While statistical uncertainties in the clustering analysis prevent us from performing a similar sophisticated comparison here, the consistent antibias on small scales for the X-ray AGNs suggests that they may also preferentially reside in central galaxies.

\subsubsection{IR AGNs}

For the IR-selected AGNs, the cross-correlation with AGES galaxies is weaker and has a flatter slope than for radio or X-ray AGNs. The estimated dark matter halo masses are $M_{\text {halo }} \lesssim 10^{12} h^{-1} M_{\odot}$. These halo mass estimates are uncertain because at these redshifts and halo masses, $b_{\text {abs }}$ is a weak function of $M_{\text {halo }}$. However, we can conclude that IR AGNs typically reside in halos that are significantly smaller than those that host radio or X-ray AGNs. We also note that the reduced $\chi^{2}$ for the fit to $w_{p}\left(r_{p}\right)$ is quite low (0.4) for the IR AGNs, 
possibly because of covariance between the $w_{p}\left(r_{p}\right)$ on different scales that is not reflected in the errors on the individual points. The uncertainties in the fit parameters and the bias are derived directly from resampling, and so account for any covariance between scales.

In contrast to the radio or X-ray AGNs, optically extended IR AGNs are significantly antibiased relative to normal galaxies with similar optical properties. This suggests that the process that triggers IR-bright AGN activity depends not only on host galaxy properties but also on local environment, with AGNs preferring underdense regions. The optically extended IR AGNs are less biased (more strongly antibiased) on small scales $(0.3-$ $\left.1 h^{-1} \mathrm{Mpc}\right)$ compared to larger scales $\left(1-10 h^{-1} \mathrm{Mpc}\right)$, reflected in the flat slope of the cross-correlation function. Keeping in mind that the relative bias on the two scales differ by only $1.5 \sigma$, this may suggest that IR AGNs preferentially reside in the central galaxies of their (relatively small) dark matter halos.

\subsubsection{X-ray and IR AGNs}

Finally, we examine the AGN-galaxy cross-correlation for objects that are selected as both X-ray and IR AGNs. Owing to the limited sample size, we do not divide these sources into optically extended and unresolved subsets. The clustering parameters, bias, and characteristic $M_{\text {halo }}$ for these sources are given in Table 2 (to avoid confusion, the cross-correlation results for this sample are not shown in Figure 11). AGNs that are selected in both the IR and X-rays show essentially identical clustering to the full sample of IR-selected AGNs, with significant antibias relative to AGES galaxies. This suggests that the IR-selected AGNs are weakly clustered, regardless of whether they are selected in X-rays, and are found in smaller dark matter halos than typical X-ray AGNs (most of which are not selected with IRAC). We discuss the implications of the clustering results for all the AGN samples in Section 9.

\section{X-RAY SPECTRA AND EDDINGTON RATIOS}

In this section, we probe the properties of the accretion process in the different classes of AGNs, by measuring average Xray spectra and Eddington ratios. There is growing evidence that black hole accretion can occur in different states, with a key parameter being the Eddington ratio (e.g., Churazov et al. 2005; Merloni \& Heinz 2008; Kauffmann \& Heckman 2009), and AGNs in these different modes may play distinct roles in galaxy evolution. In addition, many AGNs are obscured by gas and dust; according to "unified models," this obscuration originates in a roughly toroidal structure that is intrinsic to the accretion flow (e.g., Antonucci 1993; Urry \& Padovani 1995). We first compute average X-ray spectra, which can be used to detect the absorption by gas or can possibly indicate different accretion modes. We then derive bolometric AGN luminosities $\left(L_{\mathrm{bol}}\right)$ for the different classes of AGNs, which we use along with the estimates of black hole masses to derive Eddington ratios.

\subsection{X-ray Stacking Analysis}

To estimate the average X-ray spectra for AGNs, we use an Xray stacking analysis. Unabsorbed, Type 1 AGNs typically have power-law X-ray spectra with $\Gamma \simeq 1.8$ (Tozzi et al. 2006), but intervening neutral gas preferentially absorbs soft X-rays and so hardens the X-ray spectrum. Alternatively, a harder Xray spectrum may indicate a different accretion mode than is typical for optically bright Seyferts or quasars. Galactic black hole binary systems show multiple X-ray spectral states with varying spectral hardness that are caused by changes in the accretion mode (for reviews, see Remillard \& McClintock 2006; Narayan \& McClintock 2008). The "low-hard" state found in black hole binaries is often interpreted as a radiatively inefficient mode (Esin et al. 1997), such as an ADAF (Narayan \& Yi 1995). Unusually hard X-ray spectra observed in an AGN (particular for a low accretion rate) may indicate a similar mode of accretion (e.g., Hopkins et al. 2009b), as has been suggested for very low Eddington AGNs in red galaxies in AGES (Brand et al. 2005).

Even for the X-ray-detected sources in our AGN sample, we cannot accurately determine spectral shapes for individual sources since most have $\leqslant 10$ counts. However, we can use Xray stacking to calculate average spectral properties for subsets of sources. Specifically, we calculate the hardness ratio, defined as $^{11}$

$$
\mathrm{HR}=\frac{H-S}{H+S},
$$

where $H$ and $S$ are the observed counts in the hard (2-7 keV) and soft $(0.5-2 \mathrm{keV})$ bands, respectively.

The stacking procedure is described in Section 5.1 of Hickox et al. (2007). Stacked counts are defined as the number of (background-subtracted) photons detected within $r_{90}$ from the source position, where $r_{90}$ is an approximation of the $90 \%$ pointspread function (PSF) energy encircled radius at $1.5 \mathrm{keV}$, and varies as

$$
r_{90}=1^{\prime \prime}+10^{\prime \prime}\left(\theta / 10^{\prime}\right)^{2} .
$$

We subtract a small background of 3.0 count s ${ }^{-1} \mathrm{deg}^{-2}$ source $^{-1}$ in the $0.5-2 \mathrm{keV}$ band and 5.0 count $\mathrm{s}^{-1} \mathrm{deg}^{-2}$ source $^{-1}$ in the $2-7 \mathrm{keV}$ band (note that we do not include sources found in 11 pointings with high flaring background; see Section 5.1 of Hickox et al. 2007). In order to maximize the number of source counts, we do not limit the stacking to the central $6^{\prime}$ around the pointing center for each observation (unlike in Hickox et al. 2007). Count uncertainties are calculated using the approximation $\sigma_{X}=\sqrt{X+0.75}+1$, where $X$ is the number of counts in a given band (Gehrels 1986). Uncertainties in HR are derived by propagating these count rate errors.

To estimate an effective power-law photon index $\Gamma$, we use PIMMS to calculate the HR that would be observed for different values of $\Gamma$, given the ACIS Cycle 4 on-axis response function. For the small Galactic $N_{\mathrm{H}}$ toward this field $\left(\sim 10^{20} \mathrm{~cm}^{-2}\right)$, the relationship between $\mathrm{HR}$ and $\Gamma$ can be approximated by

$$
\mathrm{HR}=0.29-0.46 \Gamma \text {. }
$$

It is possible that a few very bright sources can dominate our estimates of the average HR, so we also calculate HR for only those sources with less than 20 counts. The average HR values and the corresponding $\Gamma$ estimates are given in Table 3. As a check, we perform the same analysis for the X-ray-detected sources using the observed counts in the Kenter et al. (2005) catalog (rather than the full stacking procedure of Hickox et al. 2007), and obtain essentially identical results.

As shown in Table 3, X-ray and IR AGNs with unresolved optical counterparts have relatively soft average spectra, with $\Gamma \simeq 1$.6. The bright optical emission in these sources suggests that the nucleus is relatively unobscured by dust, and their Xray spectra are typical of X-ray AGNs that have little intervening absorbing gas. Optical obscuration and X-ray absorption are strongly correlated for most AGNs (Tozzi et al. 2006), so

\footnotetext{
11 Chandra Proposer's Observatory Guide, http://cxc.harvard.edu/proposer/POG/
} 
Table 3

X-Ray Hardness Ratios and Spectral Shapes

\begin{tabular}{|c|c|c|c|c|c|}
\hline \multirow[t]{2}{*}{ Subset } & \multirow[t]{2}{*}{$N_{\text {src }}{ }^{\mathrm{a}}$} & \multicolumn{2}{|c|}{ All } & \multicolumn{2}{|c|}{$<20$ counts } \\
\hline & & HR & $\Gamma^{\mathrm{b}}$ & HR & $\Gamma^{\mathrm{b}}$ \\
\hline \multicolumn{6}{|c|}{ All AGNs } \\
\hline Radio & 103 & $-0.44 \pm 0.04$ & $1.6 \pm 0.1$ & $-0.42 \pm 0.10$ & $1.5 \pm 0.2$ \\
\hline X-ray & 318 & $-0.34 \pm 0.01$ & $1.4 \pm 0.1$ & $-0.24 \pm 0.02$ & $1.1 \pm 0.1$ \\
\hline IR & 198 & $-0.34 \pm 0.02$ & $1.4 \pm 0.1$ & $-0.11 \pm 0.04$ & $0.9 \pm 0.1$ \\
\hline \multicolumn{6}{|c|}{ AGNs (Galaxies) } \\
\hline Radio & 95 & $-0.37 \pm 0.06$ & $1.4 \pm 0.1$ & $-0.36 \pm 0.11$ & $1.4 \pm 0.2$ \\
\hline X-ray & 212 & $-0.21 \pm 0.02$ & $1.1 \pm 0.1$ & $-0.15 \pm 0.03$ & $1.0 \pm 0.1$ \\
\hline IR & 114 & $-0.10 \pm 0.04$ & $0.8 \pm 0.1$ & $0.11 \pm 0.06$ & $0.4 \pm 0.1$ \\
\hline \multicolumn{6}{|c|}{ AGNs (Unresolved) } \\
\hline Radio & 8 & $-0.51 \pm 0.06$ & $1.7 \pm 0.1$ & $-0.71 \pm 0.27$ & $2.2 \pm 0.6$ \\
\hline X-ray & 106 & $-0.46 \pm 0.02$ & $1.6 \pm 0.1$ & $-0.41 \pm 0.03$ & $1.5 \pm 0.1$ \\
\hline IR & 84 & $-0.44 \pm 0.02$ & $1.6 \pm 0.1$ & $-0.31 \pm 0.05$ & $1.3 \pm 0.1$ \\
\hline
\end{tabular}

Notes.

${ }^{\text {a }}$ Number of sources in stacked sample; excludes sources in Chandra exposures with high background.

${ }^{b}$ Effective power law photon index, derived from HR using Equation (14).

these sources are consistent with being a population of unobscured Seyfert galaxies. In contrast, the X-ray and IR AGNs with extended optical counterparts have significantly harder average X-ray spectra, with $0.8<\Gamma<1.4$. These hard Xray spectra may be caused by absorption by gas, with $N_{\mathrm{H}} \sim$ $10^{22} \mathrm{~cm}^{-2}$ (Hickox et al. 2007), or alternatively, these sources might have intrinsically hard X-ray spectra owing to radiatively inefficient accretion. In either case, it would be expected that the sources would have relatively weak optical emission, as observed. We conclude that the average X-ray spectra of the X-ray and IR AGNs are consistent with the properties of their optical counterparts.

Among the radio-selected AGNs, the six sources also detected in X-rays have relatively soft spectra with $\mathrm{HR} \simeq-0.4$, corresponding to a typical AGN spectrum with little X-ray absorption. However, the radio AGNs that are not individually detected in X-rays have a softer stacked spectrum; the average number of background-subtracted counts per source are $0.41 \pm$ 0.08 in the $0.5-2 \mathrm{keV}$ band and $0.10 \pm 0.04$ in the $2-7 \mathrm{keV}$ band. If all this flux comes from the AGN, it corresponds to a soft spectrum with $\Gamma \simeq 1$.9. The lack of hard X-ray flux in these sources provides an upper limit to the contribution from an AGN, assuming $\Gamma=1.8$. The average AGN flux for these radio galaxies must be $S_{0.5-7 \mathrm{keV}}<2.1 \times 10^{-15} \mathrm{erg} \mathrm{cm}^{-2} \mathrm{~s}^{-1}(3 \sigma$ upper limit). For the range in redshifts observed for the radio AGNs, this corresponds to an upper limit on $L_{X}$ of $(0.5-5) \times$ $10^{42} \mathrm{erg} \mathrm{s}^{-1}$.

\subsection{Eddington Ratios}

We further use the observed luminosities to estimate Eddington ratios, which can provide insights as to the accretion states of the AGNs. For a central object with mass $M_{\mathrm{BH}}$, the Eddington luminosity is given by (Shapiro \& Teukolsky 1983)

$$
L_{\mathrm{Edd}}=1.3 \times 10^{38}\left(M_{\mathrm{BH}} / M_{\odot}\right) \mathrm{erg} \mathrm{s}^{-1} .
$$

The Eddington ratio is defined as $\lambda=L_{\text {bol }} / L_{\text {Edd }}$, where $L_{\text {bol }}$ is the bolometric accretion luminosity of the system. Luminous quasars tend to have relatively high $\lambda \gtrsim 0.1$ (e.g., McLure \& Dunlop 2004; Kollmeier et al. 2006), but some Seyfert galaxies, LINERs, and low-luminosity X-ray and radio AGNs can have


Figure 14. Distributions in (a) $L_{\mathrm{bol}}$, (b) $M_{\mathrm{BH}}$, and (c) Eddington ratios for AGNs with extended optical counterparts, for radio, X-ray, and IR AGNs. The $L_{\mathrm{bol}}$ and $L_{\text {bol }} / L_{\text {Edd }}$ estimates for the X-ray undetected radio AGNs show only upper limits derived from X-ray stacking (Section 8.1). See the text in Section 8.2 for details. Radio, X-ray, and IR AGNs have progressively smaller typical black hole masses and higher Eddington ratios.

(A color version of this figure is available in the online journal.)

$\lambda \lesssim 10^{-2}$, and even as low as $\lambda \sim 10^{-8}$ (e.g., Ho 2002; Soria et al. 2006; Sikora et al. 2007).

We calculate a rough estimate of $\lambda$ for the different classes of AGNs with extended optical counterparts (for which we can estimate the galaxy stellar mass, and thus $M_{\mathrm{BH}}$ ). To estimate $L_{\text {bol }}$ for X-ray AGNs, we scale from the observed $L_{\mathrm{X}}$. For radio AGNs that are not detected in X-rays, we use the X-ray stacking results (Section 8.1) to derive approximate upper limits on the AGN luminosity. We take the $3 \sigma$ upper limit on the average nuclear $0.5-7 \mathrm{keV}$ flux, and derive the corresponding upper limit on the luminosity for each source.

We next obtain $L_{\text {bol }}$ by scaling from $L_{\mathrm{X}}$. We first convert $L_{\mathrm{X}}$ from the $0.5-7$ band to the $0.5-8 \mathrm{keV}$ band by multiplying by 1.1 , which is roughly valid for power-law spectra with photon index $0.8<\Gamma<1.8$. We then convert to $L_{\text {bol }}$ using the luminositydependent bolometric corrections of Hopkins et al. (2007), which are in the range $\mathrm{BC}_{0.5-8 \mathrm{keV}} \simeq 10-20$. This correction assumes that the intrinsic broadband SED of these AGNs is similar to that of unobscured quasars. If instead the source has an SED that is more dominated by X-ray emission, this correction may overestimate $L_{\text {bol }}$ (e.g., Vasudevan \& Fabian 2007). The distributions in $L_{\mathrm{bol}}$ for the X-ray sources (and limits for the radio AGNs not detected in X-rays) are shown in Figure 14(a). 
For IR AGNs that are not detected in X-rays, $L_{\text {bol }}$ is derived from the rest-frame $4.5 \mu \mathrm{m}$ luminosity. Using the Hopkins et al. (2007) scaling somewhat overestimates $L_{\mathrm{bol}}$, compared to the values derived from the X-rays (for those sources that are both Xray and IR AGNs). This may be owing to the fact that the host galaxy can contribute some of the observed IR flux. We therefore decrease the IR bolometric corrections by a factor of 2 so that $L_{\mathrm{bol}}$ derived from the $\mathrm{X}$-rays and IR are consistent, and use these corrections for all the IR AGNs. The distributions in $L_{\text {bol }}$ for the IR AGNs are also shown in Figure 14(a).

For an estimate of $M_{\mathrm{BH}}$, we first determine the bulge luminosity in the $B$ band $\left(L_{B, \text { bul }}\right)$ and then scale, using the relation of Marconi \& Hunt (2003), between $L_{B \text {,bul }}$ and $M_{\mathrm{BH}}$. We derive the absolute $B$-band magnitude of the galaxy, $M_{B}$, by adding the rest-frame ${ }^{0.1}(g-r)$ color to the evolution-corrected absolute magnitude in the ${ }^{0.1} r$ band (Section 3.3), and then subtracting $0.1 \mathrm{mag}$ for the small conversion between the ${ }^{0.1} \mathrm{~g}$ and $B$ bands. We then calculate $L_{B}$ (in $L_{\odot}$ ), taking the absolute magnitude of the Sun in the $B$ band to be 5.48 .

We further require an estimate of the ratio of bulge to total luminosity in the $B$ band, $(B / T)_{B}$, which can vary from less than 0.1 for Scd galaxies to 1 for ellipticals. Here, we use the morphological analysis of the Millennium Galaxy Catalog (MGC) by Allen et al. (2006), who calculated structural parameters (including bulge and disk luminosities) for a large sample of galaxies. Using a subset of 8243 galaxies from the MGC with $-24<M_{B}<-18$ (roughly the magnitude distribution of our AGN host galaxies), we calculate the average $(B / T)_{B}$ in bins of $u-r$ color. $(B / T)_{B}$ varies from $\simeq 0.1$ at $u-r=1$ (the bottom of the blue cloud) to $\sim 0.5$ at $u-r=3$ (the top of the red sequence), with a variance of $\Delta(B / T)_{B} \sim 0.2$ in each color bin. Using this relation and the rest-frame ${ }^{0.1}(u-r)$ colors (making a slight correction of 0.1 mag to convert to $u-r$ ), we derive $(B / T)_{B}$, and thus $L_{B \text {,bul }}$, for the AGN hosts.

Finally, we derive $M_{\mathrm{BH}}$ from $L_{B \text {, bul }}$ using the relation of Marconi \& Hunt (2003) for their "Group 1" sample of galaxies. The corresponding distributions in $M_{\mathrm{BH}}$ and $\lambda$ are shown in Figures 14(b) and (c). Owing to the scatter in the above relations, these $M_{\mathrm{BH}}$ estimates may be uncertain by as much as $0.5 \mathrm{dex}$ for individual galaxies, but we expect the average distribution to be accurate to within $\sim 0.2$ dex. The method for measuring $M_{\mathrm{BH}}$ likely introduces a further systematic factor of $\sim 0.3-0.5$ dex, but this does not affect the relative distributions in $M_{\mathrm{BH}}$ and $\lambda$ for the different samples, which are primarily of interest here.

As a check on these $M_{\mathrm{BH}}$ estimates, we derive a rough SMBH mass function from the AGES galaxies and compare with those derived by previous works. For galaxies with $M_{\mathrm{BH}} \lesssim 10^{9} M_{\odot}$ the optical luminosity is low enough that they are not detected out to $z=0.8$. To account for this, we use the observed distribution of black hole masses with redshift to estimate the typical volume probed for sources as a function of $M_{\mathrm{BH}}$. Including the galaxy sampling weights (Section 3.1), we derive the mass function $\phi\left(M_{\mathrm{BH}}\right)$. We directly compare this to local black hole mass functions compiled by Lauer et al. (2007), and find that they closely agree (Figure 15), suggesting that on average our $\mathrm{BH}$ mass estimates are reasonably accurate. We note that we can directly compare our $\mathrm{BH}$ mass function for objects at $0.25<z<0.8$ to $\phi\left(M_{\mathrm{BH}}\right)$ derived locally, because the highmass end of the $\mathrm{BH}$ mass function has evolved only slightly since the epochs probed by the typical AGES galaxies. The observed AGN “downsizing” (e.g., Hasinger et al. 2005; Barger et al. 2005) implies that $\mathrm{BH}$ growth at $z<0.5$ is dominated by relatively small black holes, so that the change in the $\mathrm{BH}$ mass function from $z \sim 0.5$ to $z=0$ is only 0.2 dex at $M_{\mathrm{BH}} \sim$

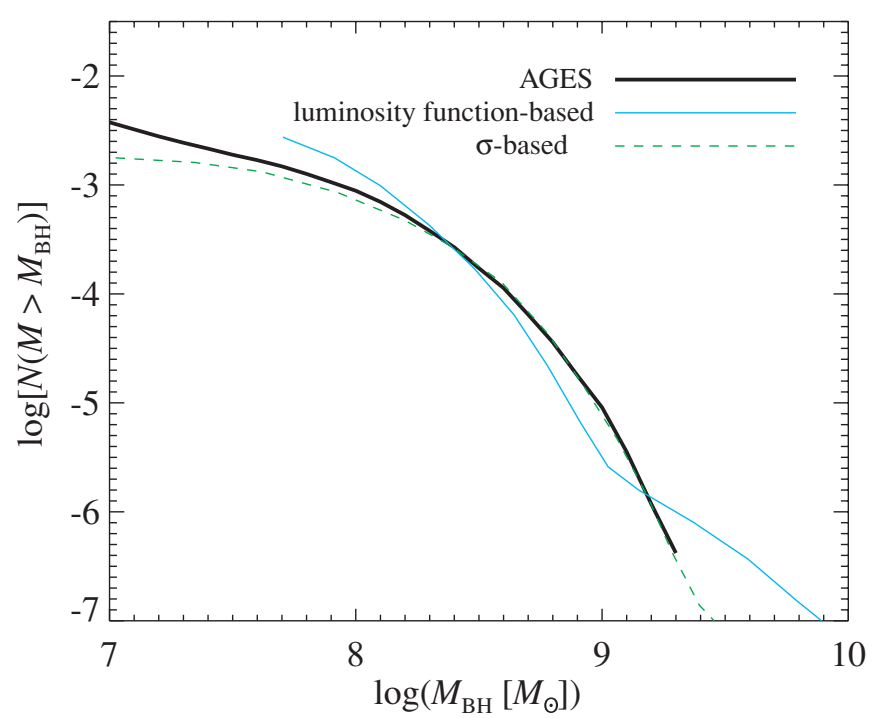

Figure 15. Black hole mass function for $0.25<z<0.8$, derived from $M_{\mathrm{BH}}$ measurements described in Section 8.2, compared to local mass function estimates in the literature. The thick black line shows $\phi\left(M_{\mathrm{BH}}\right)$ derived from the AGES data, including rough completeness corrections. The solid cyan and dashed green lines show local estimates of $\phi\left(M_{\mathrm{BH}}\right)$ compiled in Figure 11 of Lauer et al. (2007), derived from luminosity functions and distributions in bulge stellar velocity dispersions, respectively. Our rough estimates of $M_{\mathrm{BH}}$ give a BH mass function that is similar to those derived from other methods.

(A color version of this figure is available in the online journal.)

$10^{7} M_{\odot}$, and negligible for $M_{\mathrm{BH}}>10^{8} M_{\odot}$ (Merloni \& Heinz 2008).

Figure 14(c) shows that the different classes of AGNs have significantly different characteristic Eddington ratios. X-ray AGNs have a wide range of $10^{-3} \lesssim \lambda \lesssim 1$, while the IR AGNs have higher Eddington ratios, almost all having $\lambda \gtrsim 10^{-2}$. The $\mathrm{X}$-ray undetected radio AGNs have very low upper limits on $\lambda$, with most sources having $\lambda<10^{-3}$. Although these estimates of $\lambda$ are highly uncertain, particularly for individual galaxies, we can conclude that the population of X-ray AGNs extends to lower Eddington ratios than that of the IR AGNs, while the radio AGNs have very low Eddington ratios.

Finally, we note that the sources that are selected as both Xray and IR AGNs have a distribution in $\lambda$ that is almost identical to that of the full IR AGN population. The median values of $\lambda$ for the IR AGNs that are detected and undetected in X-rays differ by only 0.05 dex. We note as well that the clustering of the $\mathrm{X}$-ray and IR AGNs is essentially identical to that for the full IR AGN sample (Section 7.2.4), and that the stacked X-ray flux from the X-ray undetected IR AGNs is consistent with having lowlevel X-ray emission (as shown in Appendix B). Therefore, the IR-selected sources may represent a single coherent population of AGNs, distinct from typical X-ray AGNs, most of which are not selected with IRAC.

\section{DISCUSSION}

In this paper, we have used observations across a broad range of wavelengths together with spectroscopic redshifts to provide a general picture of the populations of accreting SMBHs, their host galaxies, their environments, and their accretion modes at moderate redshifts $(0.25<z<0.8)$. As discussed in Section 1, there is evidence for a connection between accretion onto SMBHs and the evolution of their host galaxies. Therefore, the properties of AGN and their hosts that we observe at $z \sim 0.5$ provide a "snapshot" in the co-evolution of these objects over cosmological time. 


\subsection{A Simple Model of AGN and Galaxy Evolution}

In this section, we present a simple "cartoon" model of AGN and host galaxy evolution that explains, in broad terms, the observations described in this paper. We base this model on four ideas that have received much discussion in the literature on AGN and galaxy evolution, and are supported by considerable observational and theoretical evidence.

1. In the early universe, galaxies initially form as systems that are rich in gold gas and have rotation-dominated dynamics (although they can be clumpier and more turbulent than present-day disk galaxies). This scenario is supported by observations of high-redshift galaxies (e.g., Ravindranath et al. 2006; Elmegreen et al. 2007) as well as theoretical arguments for the dissipational collapse of cold clouds (e.g., White \& Rees 1978; Fall \& Efstathiou 1980), and detailed numerical models (e.g., Robertson et al. 2004, 2006; Hopkins et al. 2009a).

2. Optical quasars are found in dark matter halos with a characteristic mass of $M_{\text {halo }} \sim 3 \times 10^{12} M_{\odot}$ that remains effectively constant with redshift. In large surveys, the bias of quasars is observed to increase with redshift, consistent with this characteristic parent dark matter halo mass (Porciani et al. 2004; Croom et al. 2005; Porciani \& Norberg 2006; Coil et al. 2007; Myers et al. 2007; Shen et al. 2007; da Ângela et al. 2008; Padmanabhan et al. 2009). Quasars represent phases of accretion at high Eddington ratios $(\lambda>0.1$; McLure \& Dunlop 2004; Kollmeier et al. 2006), in which massive black holes accrete the bulk of their final mass (e.g., Yu \& Tremaine 2002; Shankar et al. 2009; Yu \& Lu 2008).

3. The processes that fuel quasars are also responsible for the creation of stellar bulges. There is evidence that the quasar phase is often preceded by dust-obscured phases of very rapid star formation, which would be manifest observationally as ultraluminous infrared galaxies (ULIRGs) or submillimeter galaxies (SMGs; Sanders \& Mirabel 1996; Smail et al. 1997). The process that disturbs the galaxy and fuels the star formation and accretion can also likely create a dynamically hot stellar bulge. Possible physical processes include major mergers of gas-rich galaxies (e.g., Kauffmann \& Haehnelt 2000; Springel et al. 2005; Hopkins et al. 2006b) and disk instabilities (e.g., Mo et al. 1998; Bower et al. 2006; Genzel et al. 2008)

4. After the creation of the stellar bulge, the star formation in a galaxy is quenched on a relatively short timescale. The quenching can occur because the available gas gets used up in starbursts, or because the dark matter halo has increased in mass so that the gas in the system is heated to the high virial temperature of the halo and cannot cool efficiently (e.g., Rees \& Ostriker 1977; Birnboim \& Dekel 2003). Feedback from an AGN may also help to blow away gas and quench star formation (e.g., Hopkins et al. 2006b). Further mechanical feedback (likely also from an AGN) prevents subsequent gas cooling and keeps the system "red and dead" (e.g., Churazov et al. 2005; Bower et al. 2006; Croton et al. 2006; Khalatyan et al. 2008).

These ideas imply a relatively straightforward evolutionary sequence for massive galaxies and their central black holes. Most aspects of this picture have been addressed in much more detail elsewhere (e.g., Kauffmann \& Haehnelt 2000; Springel et al. 2005; Croton et al. 2006; Bower et al. 2006; Monaco et al. 2007; Hopkins et al. 2008a, 2008b; Somerville et al. 2008); here, we present only a simple overview and show that it is generally consistent with our results. Comparisons of the observations with more detailed model predictions will be left for future work.

\subsection{Evolutionary Sequence}

In our simple picture, the sequence of AGN and galaxy evolution begins in the early universe $(z \gtrsim 6)$, where galaxies are formed as gas-rich, rotation-dominated systems and contain small "seed" central black holes. As their parent dark matter halos grow up to masses $\sim 10^{12} M_{\odot}$, these galaxies retain their general morphological and dynamical properties, including flattened morphologies, small stellar bulges, and small central black holes. This growth of these galaxies occurs through the accretion of further cold gas or through minor mergers (with mass ratios $>3: 1$ ) with other gas-rich systems (Robertson et al. 2006; Hopkins et al. 2009a). As the galaxies grow, their dark matter halos also increase in mass through the hierarchical growth of structure. The largest halos grow fastest, so that the most massive overdensities at high redshift remain the most massive at lower redshift, and so on for smaller masses (e.g., Press \& Schechter 1974; Lacey \& Cole 1993).

When a galaxy's parent dark matter halo reaches a critical $M_{\text {halo }}$ between $10^{12}$ and $10^{13} M_{\odot}$, a dramatic event occurs that triggers luminous quasar activity and thus rapid growth of the central black hole, as well as producing a dynamically hot stellar system (the classical bulge). One candidate for such an event is the major merger of two gas-rich galaxies; the characteristic quasar halo mass of $M_{\text {halo }} \sim 3 \times 10^{12} M_{\odot}$ corresponds to the small group environment where major mergers of galaxies with masses $\sim M_{*}$ are most common (Hopkins et al. 2008b). Models of mergers appear to naturally produce many observed properties of quasars and the resulting spheroidal remnants, as well as their abundance and redshift evolution (Kauffmann \& Haehnelt 2000; Springel et al. 2005; Hopkins et al. 2008b), and there is evidence for merger remnants hosting individual quasars (e.g., Riechers et al. 2008; Aravena et al. 2008).

However, in addition to mergers, other types of events such as disk instabilities (e.g., Bower et al. 2006) may also possibly trigger efficient accretion onto the black hole and grow the stellar bulge. We also note that other processes, such as the accretion of recycled gas from evolved stars (Ciotti \& Ostriker 2007) may trigger a quasar after the formation of the bulge. In the context of our observations at $z \sim 0.5$, the details of the triggering mechanism are relatively unimportant. It is sufficient only that the primary mechanism for quasar accretion and the growth of the stellar bulge occur together at a characteristic dark matter halo mass, at high redshift for the largest overdensities, and at progressively later times for smaller and smaller overdensities.

After the event that builds the bulge and grows the central black hole, star formation in the galaxy must be quenched in order to produce the observed population of passively evolving, bulge-dominated galaxies with old stellar populations that are found on the red sequence (e.g., Nelan et al. 2005; Thomas et al. 2005), which is detected out to $z \sim 2$ (Zirm et al. 2008; Kriek et al. 2008). The cessation of star formation must necessarily occur after or simultaneous to the formation of the bulge, since essentially all passively evolving galaxies contain stellar bulges (Bell 2008). ${ }^{12}$ It is possible that this quenching is a direct result of the event that fuels the quasar. Processes that drive gas onto the black hole can also power intense star bursts

\footnotetext{
12 We note that the Bell (2008) result is only for central galaxies in groups or
} clusters; see Section 9.4 for a discussion. 
(as are often associated with luminous AGNs, e.g., Alexander et al. 2005; Brand et al. 2006b) that can use up the galaxy's supply of cold gas and thus halt star formation. In addition, high virial temperature of the (growing) dark matter halo can limit further accretion of cold gas and thus star formation (e.g., Rees \& Ostriker 1977; Cattaneo et al. 2006; Faber et al. 2007). Simulations suggest that the transition to this "hot halo" regime occurs at $M_{\text {halo }} \sim 10^{12}$ (e.g., Birnboim \& Dekel 2003; Dekel \& Birnboim 2006), close to the typical $M_{\text {halo }}$ for quasar activity. For our simple picture, the details of this quenching are not important; it is sufficient that soon after the formation of a massive stellar bulge, star formation in the galaxy rapidly ceases.

If the timescale for the quenching of star formation is short, the galaxy's optical spectrum will be a composite of emission from the old stellar population plus a fading contribution from the younger stars, producing "green" colors that become redder as the young stellar population evolves. The timescale for the galaxy to evolve red colors typical of early-type galaxies is $\sim 1-$ 2 Gyr (Newberry et al. 1990; Barger et al. 1996; Bower et al. 1998). This phase may be accompanied by further accretion onto the central black hole, which can evolve on a similar timescale (Hopkins et al. 2005), and may contribute to the galaxy's transition to the red sequence. Observations of local $(z<0.1)$ early-type galaxies suggest that optically detected AGN activity coincides with the decline of star formation and the transition from blue to red colors (Schawinski et al. 2007), as well as the destruction of molecular clouds (Schawinski et al. 2009a). The decline of the average black hole accretion rate from its peak in the quasar phase (when $\dot{M} \sim \dot{M}-$ Edd) can occur (with significant variability) over timescales of $\sim 1$ Gyr (Hopkins et al. 2005), finally reaching accretion rates of $\dot{M} \lesssim 10^{-2} \dot{M}_{\text {Edd }}$ or lower. At these low accretion rates (and the corresponding low luminosities), the SED of an AGN is increasingly dominated by X-ray emission (Steffen et al. 2006; Vasudevan \& Fabian 2007), and may even enter a new, radiatively inefficient mode of accretion (Churazov et al. 2005). Therefore, these fading AGNs in "green" galaxies may be most efficiently found as X-ray AGNs.

AGN activity may serve to prevent further bursts of star formation. As mentioned above, at this stage the massive stellar bulge of the galaxy will be surrounded by a halo of hot, virialized gas. While most of this gas will have very long cooling times, near the center (where the gas density is highest and cooling most efficient), gas cooling could fuel further star formation. Such cooling must be suppressed to explain the old stellar populations observed in red-sequence galaxies and the observed dearth of massive, blue star-forming galaxies at $z<1$ (Faber et al. 2007). Once cooling is suppressed, the red-sequence galaxies can further grow through "dry" mergers that would not induce further bursts of star formation.

One promising mechanism for suppressing cooling in redsequence galaxies is feedback from AGNs, which can inject mechanical energy that reheats the cooling gas (Tabor \& Binney 1993; Binney \& Tabor 1995; Churazov et al. 2002). While there is indirect evidence for such feedback from optically or Xray selected AGNs (e.g., Schawinski et al. 2007, Bundy et al. 2008), the process of AGN feedback is observed most directly in radio AGNs. In many elliptical galaxies and galaxy clusters, relativistic jets from AGNs (detected in the radio) inflate buoyant bubbles and drive shocks in the hot surrounding medium (e.g., Fabian et al. 2003; McNamara et al. 2005; Forman et al. 2007; McNamara \& Nulsen 2007). The heating rate from this process can be sufficient to balance the cooling from the hot halo, and might therefore quench star formation (Bîrzan et al. 2004; Rafferty et al. 2006; Croston et al. 2008). The Eddington ratios for accretion in these "radio-mode" outbursts are generally small $\left(\lambda \lesssim 10^{-3}\right)$, and the observed radiative power is far smaller than the inferred mechanical power of the jets (Churazov et al. 2002; Bîrzan et al. 2004). It has been proposed that these radiatively inefficient, jet-dominated outbursts may be fueled by accretion directly from the hot gas halo (in contrast to the accretion of cold gas that likely powers high-Eddington, optically luminous AGNs), and so is only possible in massive galaxies with large dark matter halos (e.g., Tasse 2008).

In summary, the simple picture described above suggests that massive galaxies undergo the following evolutionary sequence: gas-rich, star-forming, rotation-dominated system $\rightarrow$ SMG/ULIRG/quasar $\rightarrow$ "green" spheroid with declining AGN activity $\rightarrow$ red spheroid with intermittent radio AGN activity. Figure 1 of Hopkins et al. (2008b) shows a schematic of these various phases, for the case in which the quasar is triggered by major mergers. The SMG/ULIRG/quasar phase evolves on a short timescale $\left(\lesssim 10^{8} \mathrm{yr}\right)$ and occurs in dark matter halos with a characteristic $M_{\text {halo }}$ between $10^{12}$ and $10^{13} M_{\odot}$. The subsequent "green" spheroid phase evolves over somewhat longer times $(\sim 1$ Gyr $)$, while the phase of intermittent radio AGN activity can last for the subsequent lifetime of the system. A schematic of this sequence is given in Figure 16. The columns represent systems for different initial halo masses, for which the evolutionary sequence occurs at different redshifts: large dark matter halos reach the critical mass $10^{12}-10^{13} M_{\odot}$ (and thus trigger quasars) at relatively high redshifts, while for smaller systems the sequence occurs later. Therefore, at $z \sim 0.5$, objects in different stages of this sequence should be in dark matter halos of different sizes, which can be probed through their clustering.

A key element of this simple model is that quasar activity, bulge formation, and the quenching of star formation occur when dark matter halos reach the critical $M_{\text {halo }}$. Therefore, galaxies residing in halos that have not reached this mass will remain as gas-rich disks, and will not experience a luminous quasar phase and the associated rapid black hole growth. A schematic of the evolution of low halo mass systems is given in the rightmost column of Figure 16. Although these objects have never hosted luminous quasars, they can be seen as low-luminosity AGNs; various processes such as the stochastic accretion of cold gas clouds (Hopkins \& Hernquist 2006) can fuel low-level (and likely intermittent) AGN activity, while galaxy-scale processes, such as the formation of galactic bars, can enhance the gas density in the nuclear regions and preferentially help drive accretion (e.g., Ohta et al. 2007).

Accretion in low halo mass systems can proceed in a wide range of accretion rates; however, owing to the small black hole masses $\left(M_{\mathrm{BH}} \lesssim 10^{7} M_{\odot}\right)$ only the sources with relatively high accretion rates will be detected at $z \sim 0.5$ in a wide-field survey such as Boötes. The observational evidence for AGN "downsizing," in which small black holes dominate the accretion density of the universe at low redshift (e.g., Hasinger et al. 2005; Barger et al. 2005), suggests that AGNs in less-luminous, diskdominated galaxies should dominate the population of highEddington-rate AGNs at $z<0.8$. These high-Eddington AGNs would likely be identifiable by their mid-IR colors.

\subsection{Comparison to Observations}

We next compare the evolutionary scenario described above to our observational results for the host galaxies, clustering, and Eddington ratios of the different classes of AGNs. 


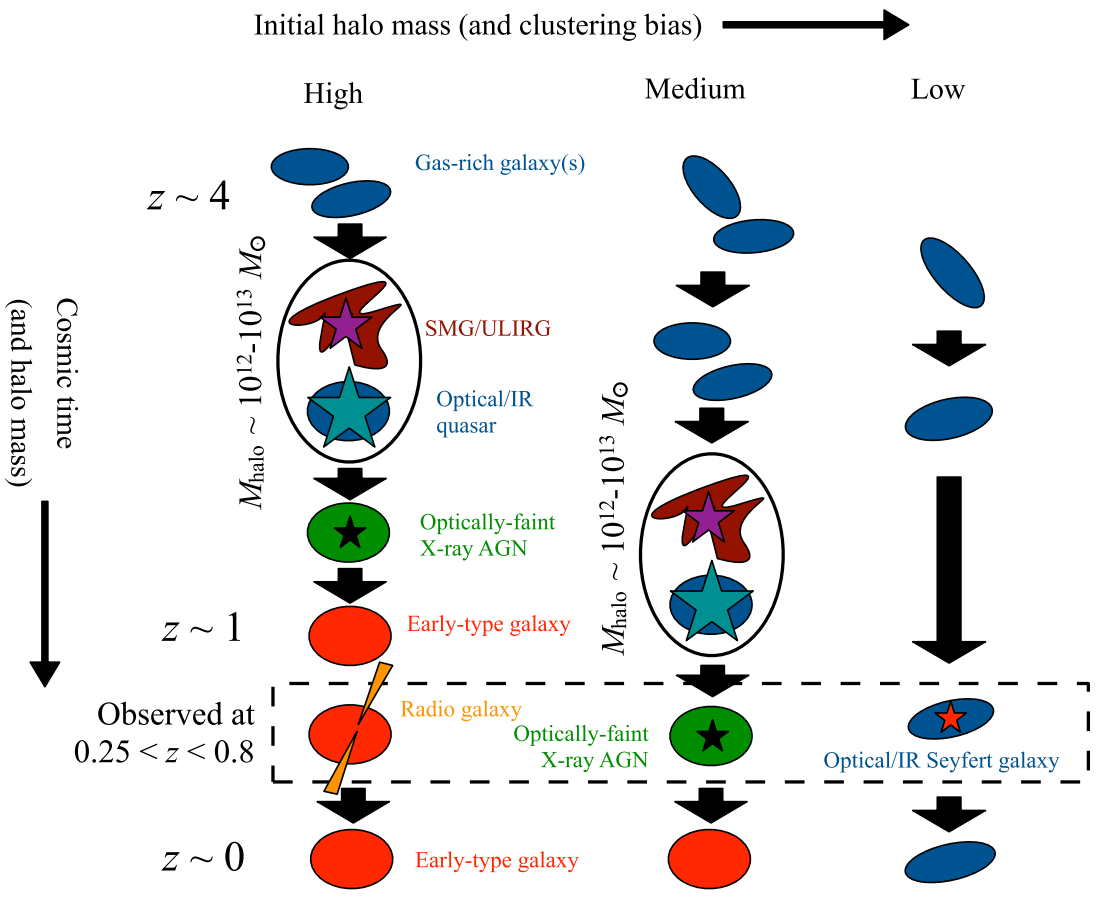

Figure 16. Schematic for a simple picture of AGN and host galaxy evolution. The picture consists of an evolutionary sequence that occurs at different redshifts for halo masses of different sizes. In this scenario, luminous AGN accretion occurs preferentially (through a merger or some secular process) when a host dark matter halo reaches a critical $M_{\text {halo }}$ between $10^{12}$ and $10^{13} h^{-1} M_{\odot}$ (this phase is indicated by the solid ovals). Once a large halo reaches this critical mass, it becomes visible as a ULIRG or SMG (owing to a burst of dusty star formation) or (perhaps subsequently) as a luminous, unobscured quasar. The ULIRG/quasar phase is associated with rapid growth of the SMBH and formation of a stellar spheroid, and is followed by the rapid quenching of star formation in the galaxy. Subsequently, the young stellar population in the galaxy ages (producing "green" host galaxy colors), and the galaxy experiences declining nuclear accretion that may be associated with an X-ray AGN. Eventually the aging of the young stars leaves a "red and dead" early-type galaxy, which experiences intermittent "radio-mode" AGN outbursts that heat the surrounding medium. For "medium" initial dark matter halos, the quasar phase and formation of the spheroid occur later than for the system with high halo mass, so that at $z \sim 0.5$ we may observe the "green" X-ray AGN phase. Even smaller halos never reach the threshold mass for quasar triggering; these still contain star-forming disk galaxies at $z \lesssim 0.8$, and we observe some of them as optical or IR-selected Seyfert galaxies. The dashed box indicates the AGN types (in their characteristic dark matter halos) that would be observable in the redshift range $0.25<z<0.8$.

(A color version of this figure is available in the online journal.)

1. Radio AGNs. Most radio AGNs in our survey at $0.25<$ $z<0.8$ are found in luminous red-sequence galaxies and are strongly clustered. In the evolutionary picture described above, radio AGNs are a key part of the late stages of massive galaxy evolution, after the bulge has formed and star formation has ceased. Therefore at $z \sim 0.5$, radio AGNs would be expected to be found primarily in massive redsequence galaxies in large dark matter halos. Accordingly, we find that radio AGNs are located in relatively large dark matter halos with $M_{\text {halo }} \sim 3 \times 10^{13} h^{-1} M_{\odot}$, corresponding to the large galaxy groups or small clusters. The radio AGNs are clustered similarly to a control sample of galaxies with matched luminosities and colors, suggesting that intermittent radio activity may be common, at least for the host galaxies and radio luminosities probed by our sample. We note that a small fraction $(\sim 20 \%)$ of the radio AGNs with extended optical counterparts are found in blue galaxies, which is contrary to this simple picture. However, some of these are likely radio-loud Seyfert galaxies and quasars, which make up $\sim 10 \%$ of those populations, and which may represent a mode of accretion more similar to radiatively efficient Seyferts than mechanically dominated radio galaxies (e.g., Merloni \& Heinz 2008; Donoso et al. 2009).

2. X-ray AGNs. X-ray AGNs in our survey at $0.25<z<0.8$ are found in host galaxies throughout the color-magnitude diagram (CMD), but the distribution peaks in the "green valley" along with a tail of bluer galaxies. The large- scale $\left(1-10 h^{-1} \mathrm{Mpc}\right)$ clustering indicates that the X-ray AGNs inhabit dark matter halos of mass $\sim 10^{13} h^{-1} M_{\odot}$, similar to the clustering for normal AGES galaxies. The Xray AGNs are unbiased relative to a control sample with matched galaxy properties, although there is a (marginally significant) antibias relative to AGES galaxies on scales less than $1 h^{-1} \mathrm{Mpc}$, suggesting that the X-ray AGNs may preferentially reside in central galaxies. In our simple evolutionary picture, central galaxies in halos with $M_{\text {halo }} \sim$ $10^{13} h^{-1} M_{\odot}$ are likely to have recently experienced the buildup of the stellar bulge and quenching of star formation, followed by a decline in the accretion rate onto the black hole. The properties of X-ray AGNs, with hosts typically showing "green" colors and with a wide range of Eddington ratios $\left(10^{-3} \lesssim \lambda \lesssim 1\right)$ are generally consistent with this picture.

3. IR AGNs. IR-selected AGNs in our observations are found in relatively low-luminosity galaxies and are weakly clustered, with characteristic $M_{\text {halo }} \lesssim 10^{12} h^{-1} M_{\odot}$. There is a significant antibias relative to a matched control sample of normal galaxies. The Eddington ratios of these sources are relatively high $\left(\lambda \gtrsim 10^{-2}\right)$, and the clustering and Eddington ratios do not depend strongly on whether the AGNs are also detected in X-rays. In our simple picture of AGN evolution, at $z<0.8$ most high-Eddington AGNs will be found in objects with small black holes, in environments that have not yet reached the critical halo mass for the 
quenching of star formation. In our observations we identify IR-selected AGNs with weakly clustered star-forming galaxies, in general agreement with this picture.

\subsection{Caveats}

The simple evolutionary scenario described above thus corresponds generally to our observational results of radio, $\mathrm{X}$-ray, and IR-selected AGNs. However, by its nature our simple model of AGN and galaxy evolution ignores many details and is subject to several important caveats. In this section, we discuss several of these issues and how they may be addressed with future observations.

1. Central versus satellite galaxies. The model described above may be only strictly valid for central galaxies, defined as the dominant galaxy in the parent dark matter halo. It is possible that the quenching of star formation can happen in different ways for satellite galaxies that fall into larger dark matter halos; ram pressure stripping, strangulation, and starvation can cause a disk galaxy to lose its cold gas and cease star formation (see van den Bosch et al. 2008, and references therein). Further, there is evidence that for satellite galaxies, star formation and AGN activity depend more strongly on the stellar mass of the galaxy than the mass of the halo (Pasquali et al. 2009). Successful models of AGN and galaxy evolution will likely need to explain the evolution of central and satellite galaxies separately. We note, however, that at least for X-ray and IR-selected AGN in our sample, the observed clustering suggests that they may preferentially reside in central galaxies (Section 7.2).

2. Critical halo mass. Our simple evolutionary model includes "critical" dark matter halo mass $M_{\text {crit }}$ between $10^{12}$ and $10^{13} M_{\odot}$ at which a galaxy undergoes luminous quasar activity and the growth of the stellar bulge. This idea is motivated by observations of quasar clustering and models of hot gaseous halos, but the details of this process are certainly more complicated than can be described by a single critical halo mass. Indeed, galaxy evolution models in which quenching occurs at a halo mass threshold may fail to reproduce the density of passively evolving galaxies at high redshift, as well as relative numbers of red and blue galaxies (Hopkins et al. 2008a). Further, evidence from the evolution of luminosity function on the red and blue sequences suggests that quenching may occur with different mechanisms, and in different mass halos (Faber et al. 2007). Quasar triggering and bulge formation probably occur by some more complex process that preferentially occurs at a given halo mass. The details of this process cannot be constrained with the observations presented here, but they could be tested in the future by more detailed work.

3. Extinction and galaxy colors. The above discussion makes note of the "green" galaxy host colors of the X-ray AGNs as evidence that these sources have undergone recent star formation. However, in the calculation of galaxy colors, we have not taken into account the effects of dust extinction intrinsic to the galaxy. It is possible that some galaxies in the "green valley" are in fact actively star-forming galaxies that are reddened by dust. In a multiwavelength study of galaxies at $0.05<z<1.5$ in the GOODS-North field, Cowie \& Barger (2008) found a significant population of galaxies (mainly Spitzer $24 \mu \mathrm{m}$ sources) that have observed colors in the "green valley," but lie in the blue cloud after correcting for extinction. However, in our sample the Xray AGNs in "green" galaxies show clustering consistent with that of typical quiescent "green" galaxies with similar colors (Section 7.2), and stronger clustering than typical blue cloud galaxies. Thus, it is unlikely that the majority of the "green" hosts of X-ray AGNs are dust-reddened blue cloud galaxies. In the future, a more detailed study of the optical-IR SEDs and average optical spectra of these objects would allow us to put more strict limits on the population of dust-reddened star-forming galaxies.

\section{SUMMARY}

In this paper, we explored the links between $\mathrm{SMBH}$ accretion, host galaxy evolution, and large-scale environment, by studying the host galaxies of AGNs detected at redshifts $0.25<z<0.8$ in the AGES survey. The AGNs were selected using observations in the radio, X-ray, and IR wavebands. Key results from this paper are as follows.

1. AGNs in the AGES sample at $0.25<z<0.8$ that are selected in different wavebands comprise generally distinct populations of sources. Radio AGNs are generally not selected in the other bands, while there is $30 \%-50 \%$ overlap between the X-ray and IR-selected AGNs.

2. The host galaxies of optically faint AGNs are significantly different between the three AGN samples. Radio AGNs are found in luminous red-sequence galaxies. X-ray AGNs are found in galaxies of all colors, with a peak in the "green valley." IR AGN hosts are relatively bluer and less luminous than those of the X-ray or radio AGNs.

3. The two-point cross-correlation between AGNs and galaxies is significantly different for the three classes of AGNs. We estimate that radio, X-ray, and IR AGNs are found in dark matter halos with characteristic masses $\sim 3 \times$ $10^{13} h^{-1} M_{\odot}, \sim 10^{13} h^{-1} M_{\odot}$, and $\lesssim 10^{12} h^{-1} M_{\odot}$, respectively.

4. X-ray and radio AGNs with extended galaxy counterparts are clustered similarly to samples matched in color, absolute magnitude, and redshift, indicating that they inhabit environments typical of their parent host galaxy populations. Conversely, IR AGNs are weakly clustered relative to a matched galaxy sample, suggesting that the process that triggers IR-bright AGN accretion depends on local environment as well as host galaxy type, and is more likely to occur in regions of lower galaxy density.

5. The average X-ray spectra of X-ray and IR AGNs are consistent with the properties of their optical counterparts. Optically bright AGNs have soft X-ray spectra with photon index $\Gamma \simeq 1.6$, typical of unabsorbed AGNs. Optically faint AGNs have harder average spectra, which likely indicates either absorption by intervening gas, or a radiatively inefficient mode of accretion.

6. Most radio AGNs in our sample have massive black holes $\left(M_{\mathrm{BH}}>10^{8} M_{\odot}\right)$ and very small Eddington ratios $\left(\lambda<10^{-3}\right)$. X-ray AGNs have smaller typical $M_{\mathrm{BH}}$ values that extend down to $\sim 10^{7} M_{\odot}$, and $\lambda$ between $10^{-3}$ and 1. IR AGNs have relatively small black holes $\left(3 \times 10^{7} \lesssim M_{\mathrm{BH}} \lesssim 3 \times 10^{8} M_{\odot}\right)$ and high Eddington ratios $\left(\lambda>10^{-2}\right)$.

7. Sources that are selected as both X-ray and IR AGNs have host galaxy properties, clustering, and Eddington ratios that are similar to those for the full IR AGN population. This 




Figure 17. Average flux profiles for subsets of galaxies and AGNs from the AGES survey, in the $B_{W}$ (top) and $I$ (bottom) bands. Fluxes are calculated in annular apertures of width $1^{\prime \prime}$ (the $x$-axis gives the outer diameter of the annulus). Shown are the profiles for normal AGES galaxies (black solid line), AGNs with extended optical counterparts (radio, orange dotted line; X-ray, green solid line; IR, red dashed line), X-ray AGNs with unresolved optical counterparts (blue dot-dashed line), and a 1".35 (FWHM) Gaussian PSF (black dotted line). The AGNs in extended sources have relatively flat flux profiles similar to normal galaxies, suggesting that they have little nuclear contamination.

(A color version of this figure is available in the online journal.)

suggests that the IR-bright AGN may represent a single population of AGNs, distinct from typical X-ray AGNs (most of which are not selected with IRAC).

8. The host galaxies, clustering, and Eddington ratios of the three classes of AGNs are generally consistent with a simple picture of AGN and galaxy evolution in which SMBH growth and the quenching of star formation occur preferentially at a characteristic dark matter halo mass $M_{\text {halo }} \sim 10^{12}-10^{13} M_{\odot}$, and are followed by a decline in the AGN accretion rate and a change to a radiatively inefficient, mechanically dominated accretion mode.

We thank our colleagues on the NDWFS, AGES, IRAC Shallow Survey, and XBoötes teams. We thank Matthew Ashby, Anca Constantin, Alexey Vikhlinin, and Steven Willner for fruitful discussions, and thank the referee, Philip Hopkins, for helpful suggestions. We also thank Martin White for sharing dark matter simulation results. The NOAO Deep Wide-Field Survey, and the research of A.D. and B.T.J. are supported by NOAO, which is operated by the Association of Universities for Research in Astronomy (AURA), Inc., under a cooperative agreement with the National Science Foundation. This paper would not have been possible without the efforts of the Chandra, Spitzer, KPNO, and MMT support staff. Optical spectroscopy discussed in this paper was obtained at the MMT Observatory, a joint facility of the Smithsonian Institution and the University of Arizona. R.C.H. was supported by an SAO Postdoctoral Fellowship, NASA GSRP Fellowship, and Harvard Merit Fellowship, and by Chandra grants GO5-6130A and GO5-6121A.



Figure 18. NDWFS I-band image of a randomly selected X-ray AGN with an extended optical counterpart at $z=0.55$. The circles show the apertures used for determining the color contamination from the nucleus; the solid blue line shows the inner $4^{\prime \prime}$ aperture, while the red dashed lines show the annulus with inner and outer radii of $3^{\prime \prime}$ and $7^{\prime \prime}$, respectively.

(A color version of this figure is available in the online journal.)

\section{APPENDIX A}

\section{ESTIMATING NUCLEAR CONTAMINATION FOR AGN HOST GALAXIES}

Here, we describe in detail our method to estimate the contribution of active nuclei to the optical colors of X-ray and radio-selected AGNs. For unobscured, optically bright Seyfert galaxies and quasars, the optical emission from the nucleus is generally significantly bluer than the host galaxy, so that the combined AGN plus galaxy is bluer than the galaxy alone. For our study of the colors and luminosities of AGN host galaxies (Figure 9), we have chosen sources that are optically extended (and so not dominated by a central point source), but the nucleus may make some contribution to their integrated optical flux.

To test for nuclear contamination, we use the optical imaging to calculate the optical flux and color profiles of the different types of sources. Here, we use aperture photometry derived using the technique of Brown et al. $(2007,2008)$, with the $B_{W}$, $R$, and $I$-band photometry smoothed to a common PSF with FWHM 1.35 (to avoid PSF differences between bands), and uncertainties estimated using a Monte Carlo technique.

Evidence for some nuclear contamination is given in Figure 17, which shows the average $B_{W}$ and $I$-band flux profiles for normal galaxies, and radio, X-ray, and IR AGNs with extended optical counterparts. For comparison, we also show profiles for X-ray sources with unresolved optical morphologies (see Section 4.4), as well as the expected profile for a Gaussian PSF with FWHM 1".35, typical of the NDWFS observations. The profiles are calculated in annuli of $1^{\prime \prime}$ width. Normal galaxies have broad, extended profiles, while optically unresolved X-ray AGNs have more strongly peaked profiles indicating that they have a significant contribution from a nuclear point source. The radio AGNs have a broad average profile similar to that of quiescent galaxies, indicating that on average, they have little or no 




Figure 19. Color profiles of galaxies and AGNs in the AGES sample. Shown is $\Delta C$ (the difference in $B_{W}-I$ color between the $3^{\prime \prime}-7^{\prime \prime}$ annulus and the central $4^{\prime \prime}$ aperture) vs. rest-frame color ${ }^{0.1}(u-r)$, as derived in Section 3.3. The contours and points represent normal galaxies, while the open circles, stars, and squares represent radio, X-ray, and IR AGNs with extended optical counterparts. The bottom right panel shows the same quantities for AGNs with broad lines in their optical spectra. Optically unresolved sources are shown in dark blue, and optically extended sources are shown in light blue. In all panels, the thick lines show linear fits to $\Delta C$ vs. ${ }^{0.1}(u-r)$ for the three sets of sources (see the text for details).

(A color version of this figure is available in the online journal.)

nuclear contamination (this may be expected, given that most radio AGNs also show little nuclear activity in the X-rays or IR). In contrast, X-ray and IR-selected AGNs show a slight excess in the $B_{W}$ band over the quiescent galaxies inside a $3^{\prime \prime}$ diameter, suggesting a moderate contribution from a nuclear point source. There is only a weak excess for the X-ray and IR AGNs in the $I$ band, however, indicating that the nuclear contribution is bluer than the host galaxy. Therefore nuclear emission may affect the integrated colors of the source.

For the radio, X-ray, and IR AGNs, we wish to estimate the extent of this optical color contamination by the active nucleus, and thus produce corrected rest-frame ${ }^{0.1}(u-r)$ colors for the host galaxies alone. To this end, we compare the optical colors observed in the center of AGN-hosting galaxies to that at the outskirts, and compare these to quiescent galaxies. First, for all AGES galaxies we derive the quantity $C_{3-7}$, which is the $B_{W}-I$ color in an annular aperture of inner and outer diameters $3^{\prime \prime}$ and $7^{\prime \prime}$, respectively (Figure 18). Since the observations have a PSF of width 1".35 (FWHM), excluding the central 3" removes most of the nuclear contribution while including enough flux from the outskirts of the galaxy to derive sufficiently accurate colors. We then calculate $\Delta C$, the difference between $C_{3-7}$ and $C_{4}$ (the $B_{W}-I 4^{\prime \prime}$ aperture colors). We would expect nuclear contamination to make $C_{4}$ bluer, but to have little effect on $C_{3-7}$, so that contamination should increase $\Delta C$.

We note that the color profiles of galaxies vary with galaxy type; early-type galaxies are redder in the centers (where they are dominated by old, higher metallicity stars) compared to late-type galaxies. Figure 19 shows $\Delta C$ versus the rest-frame $0.1(u-r)$; contours and points show this distribution for normal galaxies, while stars and circles represent $\mathrm{X}$-ray and radio AGNs, respectively. For normal galaxies, $\Delta C$ increases slightly with bluer ${ }^{0.1}(u-r)$; the reddest galaxies have $\Delta C \simeq-0.1$ while the bluest have $\Delta C \simeq 0$. In contrast, the radio, X-ray, and IRAC AGNs show stronger increases in $\Delta C$ with bluer colors, indicating that some X-ray AGNs have significant nuclear emission. The solid black and dashed green, orange, and red lines show linear average fits to the trend of $\Delta C$ with $0^{0.1}(u-r)$. The trends are roughly linear; there is no significant difference if we compute the average $\Delta C$ in bins of ${ }^{0.1}(u-r)$, so for simplicity we use the linear fit.

Using these fits, we can derive the average nuclear contamination of the observed color as a function of the integrated ${ }^{0.1}(u-r)$ obtained for X-ray and radio AGN. For a given ${ }^{0.1}(u-r)$, the $B_{W}-I 4^{\prime \prime}$ color of the host galaxy $\left(C_{\mathrm{gal}}\right)$ is related to the observed $4 "$ color $\left(C_{4}\right)$ by

$$
C_{\text {gal }}=C_{4}+(\Delta C)_{\text {corr }}
$$

where $(\Delta C)_{\text {corr }}$ is a function of ${ }^{0.1}(u-r)$, and is simply the difference between the dashed green line and the solid black line in Figure 19. Including all radio, X-ray, and IR AGNs, the best linear fit gives

$$
(\Delta C)_{\text {corr }}=0.58+0.20\left[^{0.1}(u-r)\right] .
$$

We note that, as is clear in Figure 19, $(\Delta C)_{\text {corr }}$ versus ${ }^{0.1}(u-r)$ is slightly different for the three classes of AGNs, but for simplicity we use the fit that includes for all AGNs.

For normal galaxies and AGNs, the average $\Delta C$ increases slightly with redshift; if we divide the sample into sources with $0.25<z<0.5$ and $0.5<z<0.8$, the average $\Delta C$ differs by up to $\simeq 0.2$ (depending on ${ }^{0.1}(u-r)$ ). However, the change with redshift is the same for both normal galaxies and AGNs, so $(\Delta C)_{\text {corr }}$ (as a function of ${ }^{0.1}(u-r)$ ) changes by very little (less than 0.04). For simplicity, we therefore use the single parameterization for $(\Delta C)$ corr versus ${ }^{0.1}(u-r)$ (Equation (A2)) for all $0.25<z<0.8$. 




Figure 20. $K$-corrected rest-frame color ${ }^{0.1}(u-r)$ vs. observed $B_{W}-I 4^{\prime \prime}$ aperture color, for AGES main sample galaxies (points), and AGNs with extended optical counterparts: radio (circles), X-ray (stars), and IRAC (squares). Sources and broken power-law fits are shown for two redshift bins.

(A color version of this figure is available in the online journal.)

For comparison to the radio, X-ray, and IR AGNs with extended counterparts, we also derive $\Delta C$ for $\mathrm{X}$-ray sources at $0.25<z<0.8$ with broad emission lines in their AGES optical spectra, indicating that they are optically unobscured AGNs. ${ }^{13}$ These objects are shown in the lower right panel of Figure 19. Sources with unresolved morphologies (broadline, unresolved AGNs) are shown in dark blue, and those with extended morphologies are shown in light blue. The broadline, optically unresolved AGNs typically have $\Delta C \simeq 0.5$ and have ${ }^{0.1}(u-r)<1$, and they do not show a trend in $\Delta C$ with ${ }^{0.1}(u-r)$, while the optically extended sources show a similar trend to the radio, X-ray, and IR AGNs in the other four panels, although the broad-line AGNs have slightly stronger average contamination. Since most AGNs with extended galaxy counterparts have smaller $\Delta C$ and redder ${ }^{0.1}(u-r)$ than is typical

\footnotetext{
13 As mentioned in Section 1, in this paper we generally do not make use of optical spectroscopic data for selecting AGNs, because of significant selection effects. In estimating nuclear color contamination, however, these sources are useful because the spectroscopy provides evidence for some optical contribution from the AGN.
}

of optically unresolved AGNs with broad lines, we conclude that these sources are not as dominated by a nucleus (as also indicated in Figure 17, and that their colors may be robustly corrected for nuclear contamination.

To correct the colors for nuclear contamination, we use Equation (A2) to derive $(\Delta C)_{\text {corr }}$ for each source, and so determine a "corrected" $C_{4}$, then convert this to a corrected restframe color ${ }^{0.1}(u-r)$. To determine how ${ }^{0.1}(u-r)$ varies with $C_{4}$, we divide AGES main sample galaxies into seven bins of redshift from 0.25 to 0.8 , and fit the dependence of ${ }^{0.1}(u-r)$ on $C_{4}$ in each bin. A broken power-law model with a break at $C_{4}=3.3$ gives a good fit for all redshifts; examples of two fits are given in Figure 20. The relation between ${ }^{0.1}(u-r)$ and $C_{4}$ becomes shallower with increasing redshift, with slopes given by

$$
\frac{\Delta^{0.1}(u-r)}{\Delta C_{4}}= \begin{cases}0.73+1.27(z-0.70)^{2} & \left(C_{4}<3.3\right) \\ 0.33+3.17(z-0.72)^{2} & \left(C_{4} \geqslant 3.3\right) .\end{cases}
$$

We thus calculate the correction to ${ }^{0.1}(u-r)$ by multiplying $(\Delta C)_{\text {corr }}$ (from Equation (A2)) by $\Delta^{0.1}(u-r) / \Delta C_{4}$ (from Equation (A3)). The typical corrections are small, varying from $\simeq 0.3$ for the bluest AGNs, to less than 0.05 for the reddest AGNs. The effects of these nuclear contamination corrections on the host-galaxy color distribution of the AGN sample are shown in Figure 9.

\section{APPENDIX B}

\section{LIMITS ON STAR BURST CONTAMINATION OF IR-SELECTED AGN SAMPLE}

As discussed in Section 4.3, it is possible that the IRACselected AGN sample may contain some sources whose IR emission is powered by star formation instead of nuclear accretion. For the IRAC AGNs that are also detected in Xrays (roughly half of the total sample, and $\sim 1 / 3$ of those with extended optical counterparts), the X-ray emission is an unambiguous indicator of nuclear accretion. In addition, $\sim 40 \%$ of IRAC-selected AGNs show clear evidence of nuclear emission on the basis of their unresolved optical morphologies. However, for 83 (or $30 \%$ ) of the IR-selected AGNs that are optically extended and not detected in X-rays, it is useful to estimate the possible fraction of star burst contaminants.


Figure 21. (a) Histogram of source counts for the 50 IR AGNs with extended optical counterparts that are detected in X-rays. We extrapolate the roughly log-uniform distribution to lower fluxes, to model the observed counts for the X-ray undetected sources. (b) Histogram of total observed counts (source plus background) within $r_{90}$ for the 82 IR AGNs with extended optical counterparts that are inside the XBoötes coverage area and are not detected in X-rays. The red line shows the predicted counts distribution from a simple extrapolation of a log-uniform flux distribution observed for the detected sources.

(A color version of this figure is available in the online journal.) 
For this we also use X-ray emission, but are limited to X-ray stacking because the sources are not individually detected. As discussed in Section 8.1, the X-ray undetected IRAC AGNs have a high average luminosity $\left(\left\langle L_{X}\right\rangle \sim 10^{42} \mathrm{erg} \mathrm{s}^{-1}\right)$ and hard X-ray spectrum with $\mathrm{HR}=0.21 \pm 0.21$ (or $\Gamma \simeq$ 0.2 ), characteristic of absorbed AGNs. The stacked signal is significantly harder and more luminous than the typical X-ray spectra of star burst galaxies, which have $L_{X} \lesssim 3 \times 10^{41}$ erg $\mathrm{s}^{-1}$ and $\Gamma \gtrsim 1.4$ (e.g., Ranalli et al. 2003). This indicates that most of the stacked X-ray flux from these sources is from AGNs, but there remains a possibility that an X-ray-faint population of star bursts contributes significantly to the stacked flux.

To test this possibility, we extrapolate the X-ray flux distribution for the IRAC AGNs with extended counterparts that are Xray detected, and check that the stacked signal is consistent with this extrapolation. As a constraint, we use the distribution of source counts at each IRAC AGN position. This analysis constrains not only the average X-ray flux of a sample, but also the shape of its underlying flux distribution (for a detailed discussion of this technique, see Hickox \& Markevitch 2007).

The X-ray flux distribution for the 50 IRAC-selected AGNs with extended counterparts that are detected in X-rays is roughly log-uniform (Figure 21(a)). The IR luminosity and redshift distributions of the X-ray-detected sources are similar to those of their X-ray undetected counterparts, so it is reasonable to expect that the shape of the X-ray flux distribution of the IRAC AGNs may extend to fainter fluxes. We therefore create a log-uniform model flux distribution for the $82 \mathrm{X}$-ray undetected IRAC AGNs that have extended optical counterparts and lie within the $\mathrm{X}$-ray coverage area. The model sources have fluxes from 0.02 to 4 count source ${ }^{-1}$ ( 29 sources dex $^{-1}$ in flux). We randomly assign the model fluxes to each source in the sample.

We next calculate an estimated background X-ray flux from each undetected IRAC AGN, given the average background surface brightness in the 0.5-7 keV band (Section 8.1), and the area of the extraction aperture (given by the $90 \%$ energy encircled radius). The background varies from 0.01 to 0.1 count source ${ }^{-1}$. Adding the background to the flux from the model flux distribution, we calculate a predicted distribution of source counts at the IRAC AGN positions. We repeat this calculation 10 times, with different permutations of the source and background fluxes, and average the results to produce a model counts distribution.

This model distribution shows remarkably good agreement with the total number of observed source counts and their distribution, as shown in Figure 21(b) (note that the model is a direct extrapolation of the observed flux distribution, and has not been scaled to fit the observed counts). The X-ray emission from the undetected IRAC AGNs is therefore fully consistent with an extrapolation of the observed flux distribution for the Xray-detected sources.

Of course, there remains the possibility of a population of star-forming galaxies at the faint end of the sample. To put limits on such a population, we repeat the above calculation but replace the faintest model AGNs with star burst galaxies, with X-ray fluxes of 0.05 counts source ${ }^{-1}$ in $5 \mathrm{ks}$ (corresponding to $L_{X} \sim 10^{41} \mathrm{erg} \mathrm{s}^{-1}$ ). We calculate the variation in the $C$ statistic (Cash 1979; see also Hickox \& Markevitch 2007) as a function of the fraction of sources replaced by star bursts. The best match to the data is achieved for the case of no star burst contamination; to $95 \%$ confidence, the non-AGN fraction must be less than $60 \%$. This implies that for the full sample of IRAC
AGNs with extended optical counterparts, the contamination is less than $40 \%$.

In addition to the $\mathrm{X}$-ray constraints on contamination, we also note that (1) these sources would have to be highly extinguished star bursts in order to have the AGN-like IRAC colors, and (2) the optically extended IR AGNs show small but significant nuclear contamination in the optical. On the whole, these facts suggest that the star burst contamination of the IRAC AGNs with extended counterparts is most likely quite small $(\lesssim 20 \%)$. This implies that among the full IRAC AGN sample (including optically unresolved sources), star burst contamination is at most $20 \%$ and more likely $\lesssim 10 \%$.

\section{REFERENCES}

Adelberger, K. L., \& Steidel, C. C. 2005, ApJ, 630, 50

Alexander, D. M., Bauer, F. E., Chapman, S. C., Smail, I., Blain, A. W., Brandt, W. N., \& Ivison, R. J. 2005, ApJ, 632, 736

Allen, P. D., Driver, S. P., Graham, A. W., Cameron, E., Liske, J., \& de Propris, R. 2006, MNRAS, 371, 2

Alonso-Herrero, A., et al. 2006, ApJ, 640, 167

Alonso-Herrero, A., et al. 2008, ApJ, 677, 127

Antonucci, R. 1993, ARA\&A, 31, 473

Aravena, M., et al. 2008, A\&A, 491, 173

Bahcall, J. N., Schmidt, M., \& Gunn, J. E. 1969, ApJ, 157, L77

Barger, A. J., Aragon-Salamanca, A., Ellis, R. S., Couch, W. J., Smail, I., \& Sharples, R. M. 1996, MNRAS, 279, 1

Barger, A. J., Cowie, L. L., Mushotzky, R. F., Yang, Y., Wang, W.-H., Steffen, A. T., \& Capak, P. 2005, AJ, 129, 578

Barmby, P., et al. 2006, ApJ, 642, 126

Basilakos, S., Georgakakis, A., Plionis, M., \& Georgantopoulos, I. 2004, ApJ, 607, L79

Becker, R. H., White, R. L., \& Helfand, D. J. 1995, ApJ, 450, 559

Bell, E. F. 2008, ApJ, 682, 355

Bertin, E., \& Arnouts, S. 1996, A\&AS, 117, 393

Best, P. N., Kauffmann, G., Heckman, T. M., Brinchmann, J., Charlot, S., Ivezić, Ž., \& White, S. D. M. 2005, MNRAS, 362, 25

Binney, J., \& Tabor, G. 1995, MNRAS, 276, 663

Birnboim, Y., \& Dekel, A. 2003, MNRAS, 345, 349

Birzan, L., Rafferty, D. A., McNamara, B. R., Wise, M. W., \& Nulsen, P. E. J. 2004, ApJ, 607, 800

Blanton, M. R. 2006, ApJ, 648, 268

Blanton, M. R., et al. 2003a, AJ, 125, 2348

Blanton, M. R., et al. 2003b, ApJ, 594, 186

Blanton, M. R., et al. 2003c, ApJ, 592, 819

Bower, R. G., Benson, A. J., Malbon, R., Helly, J. C., Frenk, C. S., Baugh, C. M., Cole, S., \& Lacey, C. G. 2006, MNRAS, 370, 645

Bower, R. G., Kodama, T., \& Terlevich, A. 1998, MNRAS, 299, 1193

Brand, K., et al. 2005, ApJ, 626, 723

Brand, K., et al. 2006a, ApJ, 641, 140

Brand, K., et al. 2006b, ApJ, 644, 143

Brodwin, M., et al. 2006, ApJ, 651, 791

Brown, M. J. I., Boyle, B. J., \& Webster, R. L. 2001, AJ, 122, 26

Brown, M. J. I., Dey, A., Jannuzi, B. T., Brand, K., Benson, A. J., Brodwin, M., Croton, D. J., \& Eisenhardt, P. R. 2007, ApJ, 654, 858

Brown, M. J. I., Dey, A., Jannuzi, B. T., Lauer, T. R., Tiede, G. P., \& Mikles, V. J. 2003, ApJ, 597, 225

Brown, M. J. I., et al. 2008, ApJ, 682, 937

Bundy, K., et al. 2008, ApJ, 681, 931

Cash, W. 1979, ApJ, 228, 939

Cattaneo, A., Dekel, A., Devriendt, J., Guiderdoni, B., \& Blaizot, J. 2006, MNRAS, 370, 1651

Churazov, E., Sazonov, S., Sunyaev, R., Forman, W., Jones, C., \& Böhringer, H. 2005, MNRAS, 363, L91

Churazov, E., Sunyaev, R., Forman, W., \& Böhringer, H. 2002, MNRAS, 332, 729 
Ciotti, L., \& Ostriker, J. P. 2007, ApJ, 665, 1038

Coil, A. L., Hennawi, J. F., Newman, J. A., Cooper, M. C., \& Davis, M. 2007, ApJ, 654, 115

Coil, A. L., et al. 2008, ApJ, 672, 153

Coil, A. L., et al. 2009, ApJ, submitted (arXiv:0902.0363)

Constantin, A., \& Vogeley, M. S. 2006, ApJ, 650, 727

Cowie, L. L., \& Barger, A. J. 2008, ApJ, 686, 72

Cresswell, J. G., \& Percival, W. J. 2009, MNRAS, 392, 682

Croom, S. M., Smith, R. J., Boyle, B. J., Shanks, T., Miller, L., Outram, P. J., \& Loaring, N. S. 2004, MNRAS, 349, 1397

Croom, S. M., et al. 2005, MNRAS, 356, 415

Croston, J. H., Hardcastle, M. J., Birkinshaw, M., Worrall, D. M., \& Laing, R. A. 2008, MNRAS, 386, 1709

Croton, D. J., et al. 2006, MNRAS, 365, 11

da Ângela, J., et al. 2008, MNRAS, 383, 565

Davis, M., \& Peebles, P. J. E. 1983, ApJ, 267, 465

Davis, M., et al. 2003, Proc. SPIE, 4834, 161

Dekel, A., \& Birnboim, Y. 2006, MNRAS, 368, 2

de Vries, W. H., Morganti, R., Röttgering, H. J. A., Vermeulen, R., van Breugel, W., Rengelink, R., \& Jarvis, M. J. 2002, AJ, 123, 1784

Donley, J. L., Rieke, G. H., Pérez-González, P. G., \& Barro, G. 2008, ApJ, 687, 111

Donley, J. L., Rieke, G. H., Pérez-González, P. G., Rigby, J. R., \& AlonsoHerrero, A. 2007, ApJ, 660, 167

Donoso, E., Best, P. N., \& Kauffmann, G. 2009, MNRAS, 392, 617

Eddington, A. S. 1913, MNRAS, 73, 359

Eisenhardt, P. R., et al. 2004, ApJS, 154, 48

Eisenstein, D. J., Blanton, M., Zehavi, I., Bahcall, N., Brinkmann, J., Loveday, J., Meiksin, A., \& Schneider, D. 2005, ApJ, 619, 178

Elmegreen, D. M., Elmegreen, B. G., Ravindranath, S., \& Coe, D. A. 2007, ApJ, 658,763

Esin, A. A., McClintock, J. E., \& Narayan, R. 1997, ApJ, 489, 865

Faber, S. M., et al. 2007, ApJ, 665, 265

Fabian, A. C., Sanders, J. S., Allen, S. W., Crawford, C. S., Iwasawa, K., Johnstone, R. M., Schmidt, R. W., \& Taylor, G. B. 2003, MNRAS, 344, L43

Fabricant, D., et al. 2005, PASP, 117, 1411

Fall, S. M., \& Efstathiou, G. 1980, MNRAS, 193, 189

Fanaroff, B. L., \& Riley, J. M. 1974, MNRAS, 167, 31P

Ferrarese, L., \& Merritt, D. 2000, ApJ, 539, L9

Forman, W., et al. 2007, ApJ, 665, 1057

Galametz, A., et al. 2009, ApJ, 694, 1309

Gebhardt, K., et al. 2000, ApJ, 539, L13

Gehrels, N. 1986, ApJ, 303, 336

Genzel, R., et al. 2008, ApJ, 687, 59

Georgakakis, A., et al. 2007, ApJ, 660, L15

Georgakakis, A., et al. 2008, MNRAS, 385, 2049

Gilli, R., et al. 2005, A\&A, 430, 811

Gilli, R., et al. 2007, A\&A, 475, 83

Glikman, E., Helfand, D. J., \& White, R. L. 2006, ApJ, 640, 579

Gorjian, V., et al. 2008, ApJ, 679, 1040

Hasinger, G., Miyaji, T., \& Schmidt, M. 2005, A\&A, 441, 417

Hawkins, E., et al. 2003, MNRAS, 346, 78

Hickox, R. C., \& Markevitch, M. 2007, ApJ, 671, 1523

Hickox, R. C., et al. 2007, ApJ, 671, 1365

Ho, L. C. 2002, ApJ, 564, 120

Ho, L. C. 2008, ARA\&A, 46, 475

Hopkins, P. F., Cox, T. J., Kereš, D., \& Hernquist, L. 2008a, ApJS, 175, 390

Hopkins, P. F., Cox, T. J., Younger, J. D., \& Hernquist, L. 2009a, ApJ, 691, 1168

Hopkins, P. F., \& Hernquist, L. 2006, ApJS, 166, 1

Hopkins, P. F., Hernquist, L., Cox, T. J., Di Matteo, T., Robertson, B., \& Springel, V. 2005, ApJ, 630, 716

Hopkins, P. F., Hernquist, L., Cox, T. J., Di Matteo, T., Robertson, B., \& Springel, V. 2006a, ApJS, 163, 1

Hopkins, P. F., Hernquist, L., Cox, T. J., \& Kereš, D. 2008b, ApJS, 175, 356

Hopkins, P. F., Hickox, R., Quataert, E., \& Hernquist, L. 2009b, MNRAS, submitted (arXiv:0901.2936)

Hopkins, P. F., Richards, G. T., \& Hernquist, L. 2007, ApJ, 654, 731

Hopkins, P. F., Somerville, R. S., Hernquist, L., Cox, T. J., Robertson, B., \& Li, Y. 2006b, ApJ, 652, 864

Jannuzi, B. T., \& Dey, A. 1999, in ASP Conf. Ser. 191, Photometric Redshifts and the Detection of High Redshift Galaxies, ed. R. Weymann, et al. (San Francisco, CA: ASP), 111

Kaiser, N. 1987, MNRAS, 227, 1

Kauffmann, G., \& Haehnelt, M. 2000, MNRAS, 311, 576

Kauffmann, G., \& Heckman, T. M. 2009, MNRAS, submitted (arXiv:0812.1224)

Kauffmann, G., Heckman, T. M., \& Best, P. N. 2008, MNRAS, 384, 953
Kauffmann, G., et al. 2003a, MNRAS, 346, 1055

Kauffmann, G., et al. 2003b, MNRAS, 341, 33

Kauffmann, G., et al. 2003c, MNRAS, 341, 54

Kauffmann, G., White, S. D. M., Heckman, T. M., Ménard, B., Brinchmann, J. Charlot, S., Tremonti, C., \& Brinkmann, J. 2004, MNRAS, 353, 713

Kenter, A., et al. 2005, ApJS, 161, 9

Khalatyan, A., Cattaneo, A., Schramm, M., Gottlöber, S., Steinmetz, M., \& Wisotzki, L. 2008, MNRAS, 387, 13

Kocevski, D. D., Lubin, L. M., Gal, R., Lemaux, B. C., Fassnacht, C. D., \& Squires, G. K. 2009a, ApJ, 690, 295

Kocevski, D. D., Lubin, L. M., Lemaux, B. C., Gal, R., Fassnacht, C. D., Lin, R., \& Squires, G. K. 2009b, ApJ, submitted (arXiv:0809.2091)

Kollmeier, J. A., et al. 2006, ApJ, 648, 128

Kriek, M., van der Wel, A., van Dokkum, P. G., Franx, M., \& Illingworth, G. D. 2008, ApJ, 682, 896

Lacey, C., \& Cole, S. 1993, MNRAS, 262, 627

Lacy, M., et al. 2004, ApJS, 154, 166

Landy, S. D., \& Szalay, A. S. 1993, ApJ, 412, 64

Lauer, T. R., et al. 2007, ApJ, 662, 808

Ledlow, M. J., \& Owen, F. N. 1996, AJ, 112, 9

Lehmer, B. D., et al. 2009, ApJ, 691, 687

Li, C., Kauffmann, G., Wang, L., White, S. D. M., Heckman, T. M., \& Jing, Y. P. 2006, MNRAS, 373, 457

Madau, P., Ferguson, H. C., Dickinson, M. E., Giavalisco, M., Steidel, C. C., \& Fruchter, A. 1996, MNRAS, 283, 1388

Magorrian, J., et al. 1998, AJ, 115, 2285

Mandelbaum, R., Li, C., Kauffmann, G., \& White, S. D. M. 2009, MNRAS, 393, 377

Marconi, A., \& Hunt, L. K. 2003, ApJ, 589, L21

McLure, R. J., \& Dunlop, J. S. 2004, MNRAS, 352, 1390

McNamara, B. R., \& Nulsen, P. E. J. 2007, ARA\&A, 45, 117

McNamara, B. R., Nulsen, P. E. J., Wise, M. W., Rafferty, D. A., Carilli, C., Sarazin, C. L., \& Blanton, E. L. 2005, Nature, 433, 45

Merloni, A., \& Heinz, S. 2008, MNRAS, 388, 1011

Miller, C. J., Nichol, R. C., Gómez, P. L., Hopkins, A. M., \& Bernardi, M. 2003, ApJ, 597, 142

Miyaji, T., et al. 2007, ApJS, 172, 396

Mo, H. J., Mao, S., \& White, S. D. M. 1998, MNRAS, 295, 319

Monaco, P., Fontanot, F., \& Taffoni, G. 2007, MNRAS, 375, 1189

Monet, D. G., et al. 2003, AJ, 125, 984

Murray, S. S., et al. 2005, ApJS, 161, 1

Mushotzky, R. 2004, in Astrophysics and Space Science Library, Vol. 308, Supermassive Black Holes in the Distant Universe, ed. A. J. Barger (Dordrecht: Kluwer), 53

Myers, A. D., Brunner, R. J., Nichol, R. C., Richards, G. T., Schneider, D. P., \& Bahcall, N. A. 2007, ApJ, 658, 85

Nandra, K., et al. 2007, ApJ, 660, L11

Narayan, R., \& McClintock, J. E. 2008, New Astron. Rev., 51, 733

Narayan, R., \& Yi, I. 1995, ApJ, 452, 710

Nelan, J. E., Smith, R. J., Hudson, M. J., Wegner, G. A., Lucey, J. R., Moore, S. A. W., Quinney, S. J., \& Suntzeff, N. B. 2005, ApJ, 632, 137

Newberry, M. V., Boroson, T. A., \& Kirshner, R. P. 1990, ApJ, 350, 585

Ohta, K., Aoki, K., Kawaguchi, T., \& Kiuchi, G. 2007, ApJS, 169, 1

Overzier, R. A., Röttgering, H. J. A., Rengelink, R. B., \& Wilman, R. J. 2003, A\&A, 405, 53

Padmanabhan, N., White, M., \& Eisenstein, D. J. 2007, MNRAS, 376, 1702

Padmanabhan, N., White, M., Norberg, P., \& Porciani, C. 2009, MNRAS, submitted (arXiv:0802.2105)

Pasquali, A., van den Bosch, F. C., Mo, H. J., Yang, X., \& Somerville, R. 2009, MNRAS, 394, 38

Peebles, P. J. E. 1993, Principles of Physical Cosmology (Princeton, NJ: Princeton Univ. Press)

Plionis, M., Rovilos, M., Basilakos, S., Georgantopoulos, I., \& Bauer, F. 2008, ApJ, 674, L5

Polletta, M. d. C., et al. 2006, ApJ, 642, 673

Porciani, C., Magliocchetti, M., \& Norberg, P. 2004, MNRAS, 355, 1010

Porciani, C., \& Norberg, P. 2006, MNRAS, 371, 1824

Prandoni, I., Parma, P., Wieringa, M. H., de Ruiter, H. R., Gregorini, L., Mignano, A., Vettolani, G., \& Ekers, R. D. 2006, A\&A, 457, 517

Press, W. H., \& Schechter, P. 1974, ApJ, 187, 425

Puccetti, S., et al. 2006, A\&A, 457, 501

Rafferty, D. A., McNamara, B. R., Nulsen, P. E. J., \& Wise, M. W. 2006, ApJ, 652,216

Ranalli, P., Comastri, A., \& Setti, G. 2003, A\&A, 399, 39

Ravindranath, S., et al. 2006, ApJ, 652, 963

Rees, M. J., \& Ostriker, J. P. 1977, MNRAS, 179, 541

Remillard, R. A., \& McClintock, J. E. 2006, ARA\&A, 44, 49 
Richards, G. T., et al. 2005, MNRAS, 360, 839

Riechers, D. A., Walter, F., Carilli, C. L., Bertoldi, F., \& Momjian, E. 2008, ApJ, 686, L9

Robertson, B., Bullock, J. S., Cox, T. J., Di Matteo, T., Hernquist, L., Springel, V., \& Yoshida, N. 2006, ApJ, 645, 986

Robertson, B., Yoshida, N., Springel, V., \& Hernquist, L. 2004, ApJ, 606, 32

Rowan-Robinson, M., et al. 2005, AJ, 129, 1183

Sadler, E. M., Jenkins, C. R., \& Kotanyi, C. G. 1989, MNRAS, 240, 591

Sadler, E. M., et al. 2007, MNRAS, 381, 211

Sánchez, S. F., et al. 2004, ApJ, 614, 586

Sanders, D. B., \& Mirabel, I. F. 1996, ARA\&A, 34, 749

Schawinski, K., et al. 2007, MNRAS, 382, 1415

Schawinski, K., Thomas, D., Sarzi, M., Maraston, C., Kaviraj, S., Joo, S.-J., Yi, S. K., \& Silk, J. 2009a, ApJ, 690, 1672

Schawinski, K., Virani, S., Simmons, B., Urry, C. M., Treister, E., Kaviraj, S., \& Kushkuley, B. 2009b, ApJ, 692, L19

Schlegel, D. J., Finkbeiner, D. P., \& Davis, M. 1998, ApJ, 500, 525

Shankar, F., Weinberg, D. H., \& Miralda Escudé, J. 2009, ApJ, 690, 20

Shapiro, S. L., \& Teukolsky, S. A. 1983, Black Holes, White Dwarfs, and Neutron Stars: The Physics of Compact Objects (New York: Wiley Interscience) Shen, Y., et al. 2007, AJ, 133, 2222

Sheth, R. K., Mo, H. J., \& Tormen, G. 2001, MNRAS, 323, 1

Sheth, R. K., \& Tormen, G. 1999, MNRAS, 308, 119

Sikora, M., Stawarz, Ł., \& Lasota, J.-P. 2007, ApJ, 658, 815

Silverman, J. D., et al. 2008, ApJ, 675, 1025

Skrutskie, M. F., et al. 2006, AJ, 131, 1163

Smail, I., Ivison, R. J., \& Blain, A. W. 1997, ApJ, 490, L5

Smith, R. E., et al. 2003, MNRAS, 341, 1311

Somerville, R. S., Hopkins, P. F., Cox, T. J., Robertson, B. E., \& Hernquist, L. 2008, MNRAS, 391, 481

Soria, R., Fabbiano, G., Graham, A. W., Baldi, A., Elvis, M., Jerjen, H., Pellegrini, S., \& Siemiginowska, A. 2006, ApJ, 640, 126

Springel, V., Di Matteo, T., \& Hernquist, L. 2005, MNRAS, 361, 776
Steffen, A. T., Strateva, I., Brandt, W. N., Alexander, D. M., Koekemoer, A. M., Lehmer, B. D., Schneider, D. P., \& Vignali, C. 2006, AJ, 131, 2826

Stern, D., et al. 2005, ApJ, 631, 163

Strateva, I., et al. 2001, AJ, 122, 1861

Tabor, G., \& Binney, J. 1993, MNRAS, 263, 323

Tasse, C. 2008, PhD thesis, Leiden Observatory, Leiden University

Thomas, D., Maraston, C., Bender, R., \& Mendes de Oliveira, C. 2005, ApJ, 621,673

Tinker, J. L., Weinberg, D. H., Zheng, Z., \& Zehavi, I. 2005, ApJ, 631, 41

Tozzi, P., et al. 2006, A\&A, 451, 457

Treister, E., et al. 2009, ApJ, 693, 1713

Ueda, Y., Akiyama, M., Ohta, K., \& Miyaji, T. 2003, ApJ, 598, 886

Urry, C. M., \& Padovani, P. 1995, PASP, 107, 803

van den Bosch, F. C. 2002, MNRAS, 331, 98

van den Bosch, F. C., Aquino, D., Yang, X., Mo, H. J., Pasquali, A., McIntosh, D. H., Weinmann, S. M., \& Kang, X. 2008, MNRAS, 387, 79

Vasudevan, R. V., \& Fabian, A. C. 2007, MNRAS, 381, 1235

Vikhlinin, A., Forman, W., Jones, C., \& Murray, S. 1995, ApJ, 451, 553

Wake, D. A., Croom, S. M., Sadler, E. M., \& Johnston, H. M. 2008, MNRAS, 391,1674

White, M. 2001, A\&A, 367, 27

White, S. D. M., \& Rees, M. J. 1978, MNRAS, 183, 341

Yang, Y., Mushotzky, R. F., Barger, A. J., \& Cowie, L. L. 2006, ApJ, 645, 68

Yee, H. K. C., \& Green, R. F. 1987, ApJ, 319, 28

York, D. G., et al. 2000, AJ, 120, 1579

Yu, Q., \& Lu, Y. 2008, ApJ, 689, 732

Yu, Q., \& Tremaine, S. 2002, MNRAS, 335, 965

Zehavi, I., et al. 2004, ApJ, 608, 16

Zehavi, I., et al. 2005, ApJ, 630, 1

Zheng, Z., Coil, A. L., \& Zehavi, I. 2007, ApJ, 667, 760

Zheng, Z., Zehavi, I., Eisenstein, D. J., Weinberg, D. H., \& Jing, Y. 2009, ApJ, submitted (arXiv:0809.1868)

Zirm, A. W., et al. 2008, ApJ, 680, 224 